\title{
ON THE COMPLEX BORDISM OF FINITE COMPLEXES. II
}

\author{
P. E. CONNER \& LARRY SMITH
}

This manuscript represents a continuation of our study begun in [6] of the internal properties of the complex bordism functor and its external applications. As in [6], [7] we are primarily concerned with the numerical invariant hom. $\operatorname{dim}_{\Omega_{*}^{U}} \Omega_{*}^{U}(X)$ of a topological space $X$, its interpretations and applications. Our study centers around our understanding of the annihilator ideal of a spherical bordism class $\alpha \in \Omega_{*}^{U}(X)$. As in [6] we represent $\alpha$ by a map

$$
h: S^{k} \rightarrow X \text {. }
$$

To study the annihilator ideal of $\alpha$ we introduce the cofibration

$$
S^{k} \stackrel{h}{\longrightarrow} X \stackrel{g}{\longrightarrow} Y
$$

which may be extended to a cofibration

$$
X \stackrel{g}{\longrightarrow} Y \stackrel{f}{\longrightarrow} S^{k+1}
$$

in the standard manner. The annihilator ideal $A(\alpha)$ of $\alpha$ may then be identified with the image of the map $f_{*}: \widetilde{\Omega}_{*}^{U}(Y) \rightarrow \widetilde{\Omega}_{*}^{U}\left(S^{k+1}\right)$ under an $\Omega_{*}^{U}$-module isomorphism of degree $k+1$. This leads us into a study of mapping spaces into spheres which we undertake with the aid of the $R R$ theorem of AtiyahHirzebruch [8], characteristic numbers and cohomology operations. These provide us with several criteria for detecting elements of $A(\alpha)$, particularly Milnor manifolds [10], and to a complete determination of hom. $\operatorname{dim}_{\Omega_{*}^{U}} \tilde{\Omega}_{*}^{U}(X)$ for 3cell complexes. The final section contains several applications of the theory to the construction of examples which compliment those of [6], [7], [11] and [13].

\section{Preliminaries on cohomology operations and characteristic numbers}

Let $M$ be a closed weakly complex manifold and $a \in H^{*}(M, k), k=Z, Z_{q}$ or $Q$. Associated to the pair $\{M, a\}$ we have characteristic numbers 


$$
\left\langle a \cup c_{\omega}(M),[M]\right\rangle \in k \text {. }
$$

Our purposes in later sections require that we have available a procedure for computing such numbers. Specifically the numbers

$$
\left\langle a \cup s_{p^{i-1}}(c)(M),[M]\right\rangle \in Z_{p}, \quad p \text { a prime },
$$

will be of concern to us, where $s_{j}(c)$ denotes the usual $s_{\omega}$-symmetric function of the Chern classes of $M$. There is a Wu-type formula for evaluating these special types of numbers, and it is our objective in this section to establish this formula. To this end we collect some facts about the Steenrod algebra.

Recollections and notations. Let $\mathscr{A}^{*}(p)$ denote the mod $p$ Steenrod algebra and $\mathscr{P}^{*}(p)$ the algebra of reduced power operations, i.e.,

$$
\mathscr{P}^{*}(p)=\mathscr{A}^{*}(p) /(\beta),
$$

where $(\beta)$ denotes the two sided ideal generated by $\beta$ (recall that $\beta=S q^{1}$ when $p=2)$.

According to Milnor [9] the dual Hopf algebra $\mathscr{P}_{*}(p)$ to $\mathscr{P}^{*}(p)$ is given by

$$
\mathscr{P}_{*}(p) \cong Z_{p}\left[\left\{\mu_{i}\right\}\right]
$$

where

$$
\operatorname{deg} \mu_{i}=2\left(p^{i}-1\right)
$$

and

$$
\nabla^{*} \mu_{k}=\sum_{i=0}^{k} \mu_{k-1}^{p i} \otimes \mu_{i}
$$

with the convention that $\mu_{0}=1$.

The "duals" to the classes $\left\{\mu_{i}\right\}$ are primitive elements in $\mathscr{P}^{*}(p)$ which may be obtained inductively by the formulas

$$
S_{i}=\left\{\begin{array}{l}
P_{p}^{1}, i=1, \\
{\left[S_{i-1}, P^{p^{i-1}}\right], \quad i>1 .}
\end{array}\right.
$$

These formulas determine corresponding unique elements $S_{i} \in \mathscr{A}^{*}(p)$ which are primitive provided that $p \neq 2$. (Recall that for $p=2, P_{2}^{1}=S q^{2}$.) Note that $\operatorname{deg} S_{i}=2\left(p^{i}-1\right)$.

The operations $S_{i}$ may be computed on two dimensional classes by means of the following formula. Let $X$ be a space and $y \in H^{2}\left(X ; Z_{p}\right)$. Then

$$
S_{i} y=y^{p^{i}} .
$$


This formula is readily established by induction. The case $i=1$ is true by inspection. For $i>1$ we have

$$
S_{i} y=\left[P^{p i}, S_{i-1}\right] y=P^{p i} S_{i-1} y-S_{i-1} P^{p i} y .
$$

Since $i>1, P^{p i} y=0$. Therefore

$$
S_{i} y=P^{p i} S_{i-1} y=P^{p i} y^{p i-1}
$$

by our inductive assumption. The Adem relation

$$
P^{p j} Z P=\left(P^{p j-1} Z\right)^{p}
$$

now yields

$$
S_{i} y=\left(P_{p}^{1} y\right)^{p i-1}=y^{p i}
$$

as desired.

Proposition 1.1. Let

$$
\Phi: H^{*}\left(B U ; Z_{p}\right) \rightarrow H^{*}\left(M U ; Z_{p}\right)
$$

denote the Thom isomorphism. Then up to a unit in $Z_{p}$,

$$
S_{p^{i-1}}(c)=\Phi^{-1} S_{i} \Phi(1)
$$

Proof. To see this recall that $H^{*}\left(\underset{\sim}{M U} ; Z_{p}\right)$ is a coalgebra over the reduced power algebra and the action of $\mathscr{P}^{*}(p)$ on the counit

$$
\mathscr{P} *(p) \rightarrow H^{*}\left(\underset{\sim}{M} ; Z_{p}\right): \quad \alpha \rightarrow \alpha \Phi(1)
$$

is a morphism of coalgebras [17]. As $S_{i} \in \mathscr{P}^{*}(p)$ is a primitive element, it follows that $S_{i} \Phi(1) \in H^{*}\left(\underset{\sim}{M U} ; Z_{p}\right)$ is also. As $\Phi$ is an isomorphism of coalgebras, $\Phi^{-1} S_{i} \Phi(1) \in H^{2 p^{i-2}}\left(B U ; Z_{p}\right)$ is primitive too. Since up to a unit in $Z_{p}$, $H^{2 j}\left(B U ; Z_{p}\right)$ contains the unique primitive $s_{j}(c)$ we must have

$$
\Phi^{-1} S_{i} \Phi(1)=\lambda s_{p^{i-1}}(c)
$$

for some $\lambda \neq 0 \in Z_{p}$ as required.

Theorem 1.2. Let $M^{m}$ be a closed weakly complex manifold and $a \in H^{m-2\left(p^{i-1)}\right.}\left(M ; Z_{p}\right)$. Then

$$
\left\langle s_{p^{i-1}}(c) \cup a,[M]\right\rangle=\left\langle-S_{i} a,[M]\right\rangle \in Z_{p}
$$

up to a unit in $Z_{p}$. 
Proof. Let

$$
c: S^{m+r} \rightarrow T M
$$

denote the standard collapse [17, Chap. II] of a sphere onto the stable normal bundle of $M^{m}$.

Lemma. Let $\alpha \in \mathscr{P}^{*}(p), \operatorname{deg} \alpha>0$, and suppose $x \in H^{*}\left(T M ; Z_{p}\right)$. Then

$$
\left\langle\Phi^{-1}(\alpha x),[M]\right\rangle=0 \in Z,
$$

where

$$
\Phi: H^{*}\left(M ; Z_{p}\right) \rightarrow H^{*}\left(T M ; Z_{p}\right)
$$

denotes the Thom isomorphism.

Proof. We have, by definition,

$$
\begin{aligned}
\left\langle\Phi^{-1} \alpha x,[M]\right\rangle & =\left\langle\alpha x, \Phi_{*}[M]\right\rangle=\left\langle\alpha x, c_{*}\left[S^{m+r}\right]\right\rangle=\left\langle c^{*} \alpha x,\left[S^{m+r}\right]\right\rangle \\
& =\left\langle\alpha c^{*} x,\left[S^{m+r}\right]\right\rangle=\left\langle 0,\left[S^{m+r}\right]\right\rangle=0,
\end{aligned}
$$

since $\mathscr{P}^{*}(p)$ acts trivially on $H^{*}\left(S^{m+r} ; Z_{p}\right)$. q.e.d.

Applying the lemma to $x=S_{i}(a \cup \Phi(1))$ we obtain

$$
\left\langle\Phi^{-1}\left[S_{i}(a \cup \Phi(1))\right],[M]\right\rangle=0 .
$$

For odd $p, S_{i} \in \mathscr{P}^{*}(p)$ is primitive and hence

$$
\begin{aligned}
S_{i}(a \cup \Phi(1)) & =S_{i} a \cup \Phi(1)+a \cup S_{i} \Phi(1) \\
& =S_{i} a \cup \Phi(1)+a \cup S_{i}(c) \cup \Phi(1)
\end{aligned}
$$

by Prop. 1.1. Hence

$$
\Phi^{-1}\left(S_{i}(a \cup \Phi(1))=S_{i} a+a \cup s_{i}(c)\right.
$$

Thus

$$
0=\left\langle S_{i} a,[M]\right\rangle+\left\langle a \cup s_{i}(c),[M]\right\rangle
$$

yielding the result.

For $p=2$ we have

$$
\Delta S_{i}=S_{i} \otimes 1+1 \otimes S_{i}+\sum_{j>0} \alpha_{j} \otimes \alpha_{r-j},
$$

where $\Delta$ is the coproduct in $\mathscr{A}^{*}(2)$ and $\alpha_{i} \in(\beta)$. Thus 


$$
S_{i}(a \cup \Phi(1))=S_{i} a \cup \Phi(1)+a \cup S_{i} \Phi(1)+\sum \alpha_{i} a \cup \alpha_{i_{-j}} \Phi(1)
$$

But

$$
\alpha_{i-j} \Phi(1)=0: j=1, \cdots, i-1,
$$

as $(\beta)$ annihilates $\Phi(1)$. Therefore

$$
S_{i}(a \cup \Phi(1))=S_{i} a \cup \Phi(1)+a \cup S^{i} \Phi(1)
$$

and we may proceed as in the case of odd primes.

\section{Mapping spaces into spheres I}

As noted in the introduction we will be concerned with complexes of the form $c X \cup_{f} S^{2 k}$ where $c X$ denotes the cone on the complex $X$ and

$$
f: X \rightarrow S^{2 k}
$$

is a continuous map. Our objective is to obtain examples of such complexes whose complex bordism module is of fairly high homological dimension and to characterize certain classes of examples where this homological dimension is not too large. Our approach to this study forces us to examine

$$
\operatorname{Im}\left\{f_{*}: \tilde{\Omega}_{*}^{U}(X) \rightarrow \tilde{\Omega}_{*}^{U}\left(S^{2 k}\right)\right\}
$$

in some detail. Let us therefore suppose that $[M, g] \in \widetilde{\Omega}_{*}^{U}(X)$. Then

$$
f_{*}[M, g]=[W] \sigma,
$$

where $[W] \in \Omega_{*}^{U}$, and $\sigma \in \tilde{\Omega}_{2 k}^{U}\left(S^{2 k}\right)$ is the canonical class. The class [W] may be obtained as follows. Observe that

$$
f_{*}[M, g]=[M, f \cdot g],
$$

and we may homotope

$$
f \cdot g: M \rightarrow S^{2 k}
$$

to a differentiable map which is $t$-regular along a point $\infty \in S^{2 k}$ without changing the class of $[M, f \cdot g] \in \tilde{\Omega}_{*}^{U}\left(S^{2 k}\right)$. We let $W=(f \circ g)^{-1}(\infty)$. Then $W \subset M$ is a submanifold of codimension $2 k$ with trivial normal bundle in $M$. The stable normal bundle of $W$ may therefore be taken as

$$
\nu_{W}=\left.\varepsilon^{2 k} \oplus \nu_{M}\right|_{W}
$$


and hence has a natural complex structure. The usual $t$-regularity argument then yields

$$
f^{*}[M, g]=[W] \cdot \sigma \in \tilde{\Omega}_{*}^{U}\left(S^{2 k}\right) .
$$

To compute the characteristic numbers of $[W]$ we appeal to the AtiyahHirzebruch $R R$ theorem [3]. To this end we denote by $\eta$ the canonical bundle over $S^{2 k}$ obtained via periodicity. Let ([5], [17]) $\nu^{i}(N) \in K^{2 i}(N)$ denote the $i^{\text {th }} K$ theory Chern class of a weakly complex manifold $N$. Then [3] for any partition $\omega$ we have

$$
\left\langle\nu^{\omega}(W),[W]\right\rangle=\left\langle f^{\prime}(\eta-1) \nu^{\omega}(M),[M]\right\rangle .
$$

Since the integral cohomology Chern numbers are special cases of the $K$-theory numbers we obtain in view of the formula [17, p. 119]

$$
\left\langle c_{\omega}(W),[W]\right\rangle=\left\langle f^{*} i \cup c_{\omega}(M),[M]\right\rangle,
$$

where

$$
i \in H^{2 k}\left(S^{2 k} ; Z\right)
$$

is the canonical class.

Let us fix the following notations to facilitate some computations:

(1) $f: X \rightarrow S^{2 k}$ is a continuous map.

(2) $d$ is the order of the cokernel of the induced map $f_{*}: H_{2 k}(X ; Z) \rightarrow$ $H_{2 k}\left(S^{2 k} ; Z\right)$.

(3) $\eta$ is the canonical bundle over $S^{2 k}$ obtained via periodicity, so that $\eta-1 \in \tilde{K}\left(S^{2 k}\right) \cong Z$ is a generator.

Theorem 2.1. With the above notations let $c \in Z, c \neq 0$. Then $\operatorname{Im}\left\{f_{*}: \widetilde{\Omega}_{*}^{U}(X)\right.$ $\left.\rightarrow \tilde{\Omega}_{*}^{U}\left(S^{2 k}\right)\right\}=c \widetilde{\Omega}_{*}^{U}\left(S^{2 k}\right)$ iff $f^{!}(\eta-1)$ is divisible by $c$ in $\tilde{K}(X) /$ torsion.

Proof. The proof will make use of the relation between complex bordism and the $Z_{2}$ graded homology theory associated to the $B U$-spectrum. There is a natural epijection $\Omega_{*}^{U}(\cdot) \rightarrow K_{*}(\cdot)$ preserving degrees mod 2 . The fundamental fact relating bordism to $K$-theory is the isomorphism $[5, \S 9]$ (see also [6, § 9])

$$
Z_{T d} \otimes_{\Omega v *} \Omega_{*}^{U}(\cdot) \cong K_{*}(\cdot)
$$

where $Z$ receives a $Z_{2}$-graded $\Omega_{*}^{U}$-module structure from the Todd genus $T d: \Omega_{*}^{U} \rightarrow Z$.

With this in mind let us suppose that

$$
\operatorname{Im}\left\{f_{*}: \tilde{\Omega}_{*}^{U}(X) \rightarrow \tilde{\Omega}_{*}^{U}\left(S^{2 k}\right)\right\}=c \tilde{\Omega}_{*}^{U}\left(S^{2 k}\right)
$$

From the diagram 


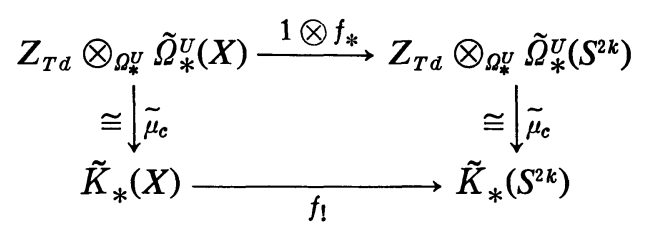

we see that

$$
\operatorname{Im}\left\{f_{!}: \tilde{K}_{*}(X) \rightarrow \tilde{K}_{*}\left(S^{2 k}\right)\right\} \not \equiv c \tilde{K}_{*}\left(S^{2 k}\right) .
$$

Consider now the universal coefficient sequences:

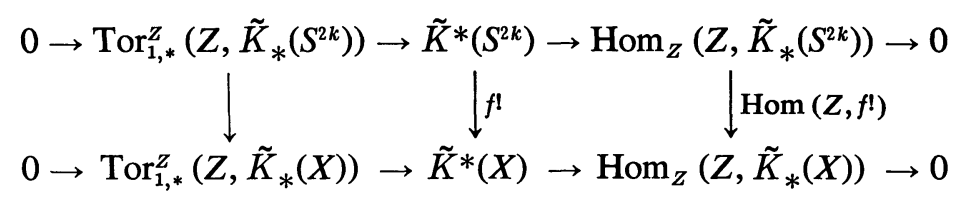

Recalling that

$$
\operatorname{Tor}_{1, *}^{Z}\left(Z, \tilde{K}_{*}\left(S^{2 k}\right)\right)=0,
$$

and

$$
\operatorname{Tor}_{1, *}^{Z}\left(Z, \tilde{K}_{*}(X)\right)
$$

is isomorphic to the Torsion subgroup of $\tilde{K}^{*}(X)$ we find that $(*)$ implies $f^{!}(\eta-1) \in \tilde{K}^{*}(X) /$ torsion is divisible by $c$.

Converseley let us suppose that $f^{!}(\eta-1)$ is divisible by $c$ in $\tilde{K}^{*}(X) /$ torsion. If $[W] \cdot \sigma \in \operatorname{Im}\left(f_{*}: \tilde{\Omega}_{*}^{U}(X) \rightarrow \tilde{\Omega}_{*}^{U}\left(S^{2 k}\right)\right\}$, then

$$
[W] \sigma=f^{*}[M, g]:[M, g] \in \tilde{\Omega}_{*}^{U}(X),
$$

and hence by our discussion of $K$-theory characteristic numbers preceeding we obtain

$$
\nu^{\omega}[W]=\left\langle g^{\prime} f !(\eta-1) \nu^{\omega}[M],[M]\right\rangle \equiv 0 \bmod c,
$$

since $f !(\eta-1)=c \xi+t$ where $t \in \bar{K}^{*}(X)$ is a torsion class. Therefore $[W] \in c \Omega_{*}^{U}$ by the Hattori-Stong theorem [5], [17], and the result follows.

Corollary 2.2. With the above notations we have

$$
\operatorname{Im}\left\{f_{*}: \tilde{\Omega}_{*}^{U}(X) \rightarrow \tilde{\Omega}_{*}^{U}\left(S^{2 k}\right)\right\}=c \tilde{\Omega}_{*}^{U}\left(S^{2 k}\right)
$$

iff $T d[W] \equiv 0 \bmod c$ for all $[W] \cdot \sigma \in \operatorname{Im} f_{*}$.

Proof. The hypotheses trivially implies that 


$$
\operatorname{Im}\left\{f_{!}: \tilde{K}_{*}(X) \rightarrow \tilde{K}_{*}\left(S^{2 k}\right)\right\}=c \tilde{K}_{*}\left(S^{2 k}\right),
$$

and converseley. However as noted above this is equivalent to $f^{!}(\eta-1) \in \tilde{K}^{*}(X) /$ torsion being divisible by $c$. The result is thus immediate from (2.1). q.e.d.

As we shall see in later sections the

$$
\operatorname{Im}\left\{f_{*}: \tilde{\Omega}_{*}^{U}(X) \rightarrow \tilde{\Omega}_{*}^{U}\left(S^{2 k}\right)\right\}
$$

can be considerably more complex than indicated in (2.1) and (2.2). One example of how this may happen is contained in the following discussion.

Let us write $d=m p$ where $p$ is a prime. We wish to investigate when

$$
\operatorname{Im}\left\{f_{*}: \tilde{\Omega}_{*}^{U}(X) \rightarrow \tilde{\Omega}_{*}^{U}\left(S^{2 k}\right)\right\} \ni\left(m\left[V^{2 p^{i-2}}\right]+d_{i}\right) \sigma,
$$

where $\left[V^{2 p^{i-2}}\right] \in \Omega_{2 p i_{-2}}^{U}$ denotes the Milnor manifold for the prime $p$, and $d_{i} \in \Omega_{2 p^{i-2}}^{U}$ is a decomposable class all of whose mod $d$ Chern numbers vanish. Note that we will always have

$$
p m\left[V^{2 p^{i-2} \sigma \in \operatorname{Im}}\left\{f_{*}: \tilde{\Omega}_{*}^{U}(X) \rightarrow \tilde{\Omega}_{*}^{U}\left(S^{2 k}\right)\right\} .\right.
$$

Recall that according to Milnor [10] a manifold [W] has the form

$$
m\left[V^{2 p^{i-2}}\right]+d_{i} \text { iff } s_{p^{i-1}}(c)[W] \equiv m p \bmod m p^{2} .
$$

Therefore according to our previous discussion

$$
\left(m\left[V^{2 p^{i-2}}\right]+d_{i} \sigma\right) \in \operatorname{Im}\left\{f_{*}: \tilde{\Omega}_{*}^{U}(X) \rightarrow \tilde{\Omega}_{*}^{U}\left(S^{2 k}\right)\right\}
$$

iff there exists $[M, g] \in \tilde{\Omega}_{*}^{U}(X)$ such that

$$
\left\langle g^{*} f^{*} i \cup s_{p^{i-1}}(c)(M),[M]\right\rangle \equiv m p \bmod m p^{2} .
$$

The following theorem is now clear.

Theorem 2.3. Let $X$ be a finite complex and

$$
f: X \rightarrow S^{2 k}
$$

a map. Suppose that

$$
f^{*} i=d a: a \in H^{2 k}(X ; Z),
$$

and $d=p m$. Then

$$
\operatorname{Im}\left\{f_{*}: \tilde{\Omega}_{*}^{U}(X) \rightarrow \tilde{\Omega}_{*}^{U}\left(S^{2 k}\right)\right\} \ni\left(m\left[V^{2 p^{i-2}}\right]+d_{i}\right) \sigma
$$

iff there exists $[M, g] \in \widetilde{\Omega}_{*}^{U}(X)$ such that 


$$
\left\langle S_{i} g^{*}(a), M\right\rangle=1 \in Z_{p},
$$

where $g^{*}(a)$ denotes the $\bmod p$-reduction of $g^{*}(a)$.

Proof. Our preceeding discussion has shown that

$$
\operatorname{Im}\left\{f_{*}: \tilde{\Omega}_{*}^{U}(X) \rightarrow \tilde{\Omega}_{*}^{U}\left(S^{2 k}\right)\right\} \ni\left(m\left[V^{2 p^{i-2}}\right]+d_{i}\right) \sigma
$$

iff there exists $[M, g] \in \tilde{\Omega}_{*}^{U}(X)$ such that

$$
\left\langle g^{*} f^{*} i \cup s_{p^{i-1}}(c)(M),[M]\right\rangle=m p \bmod m p^{2} .
$$

As we have assumed

$$
f^{*} i=d a=m p a
$$

it is clearly equivalent to show

$$
\left\langle f^{*} g^{*}(a) \cup s_{p^{i-1}}(c)(M),[M]\right\rangle \equiv 1 \bmod p
$$

or reducing $\bmod p$

$$
\left\langle f^{*} g_{*}(a) \cup s_{p^{i-1}}(c)(M),[M]\right\rangle=1 \in Z_{p} .
$$

The result is now immediate from (1.1).

Corollary 2.4. Let $X$ be a finite complex with $H_{*}(X ; Z)$ torsion free and $f: X \rightarrow S^{2 k}$. Let $f^{*} i=d a, a \in H^{2 k}(X ; Z)$, and $d=m p, p$ a prime. Then

$$
\operatorname{Im}\left\{f_{*}: \tilde{\Omega}_{*}^{U}(X) \rightarrow \tilde{\Omega}_{*}^{U}\left(S^{2 k}\right)\right\} \ni\left(m\left[V^{2 p^{i-2}}\right]+d_{i}\right) \sigma
$$

iff

$$
S_{i} a \neq 0 \in H^{*}\left(X ; Z_{p}\right),
$$

where we have written a for the mod p reduction of itself.

\section{On attaching cells I}

Let $Y$ be a finite complex and

$$
h: S^{2 k-1} \rightarrow Y
$$

a continuous map. We may then form the mapping cylinder $X=Y \cup_{h} e^{2 k}$ and obtain the cofibration

$$
Y \underset{g}{\longrightarrow} X \underset{f}{\longrightarrow} S^{2 k}
$$

In [6] we studied the interplay between $\tilde{\Omega}_{*}^{U}(Y), \tilde{\Omega}_{*}^{U}(X)$ and the annihilator 
ideal $A(\alpha) \subset \Omega_{*}^{U}$ of the bordism element $\alpha=\left[S^{2 k-1}, h\right] \in \tilde{\Omega}_{*}^{U}(Y)$. This study led to several interesting examples. In this section we propose to continue this study with the aid of the results of $\S 2$. To this end we require

Lemma 3.1. With the above notations, the annihilator ideal $A(\alpha) \subset \Omega_{*}^{U}$ of $\alpha$ is isomorphic with the image of $f_{*}: \tilde{\Omega}_{*}^{U}(X) \rightarrow \tilde{\Omega}_{*}^{U}\left(S^{2 k}\right)$ by an $\tilde{\Omega}_{*}^{U}$-module isomorphism of degree $2 k$.

Proof. One has the exact triangle

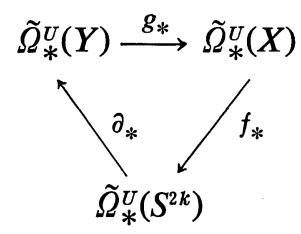

from which one sees that the asserted isomorphism is given by

$$
[M] \rightarrow[M] \cdot \sigma,
$$

where $\sigma \in \widetilde{\Omega}_{2 k}\left(S^{2 k}\right)$ is the fundamental class. q.e.d.

From Cor. 2.2 we now obtain:

Theorem 3.2. Let $h: S^{2 k-1} \rightarrow Y$ be a continuous map and $\alpha=$ $\left[S^{2 k-1}, h\right] \in \tilde{\Omega}_{*}^{U}(Y)$. Then $A(\alpha)=c \Omega_{*}^{U}$ iff $T d[W] \equiv 0 \bmod c$ for all $[W] \in A(\alpha)$. q.e.d.

The results (2.3) and (2.4) may be applied in special cases to determine when the annihilator ideal $A(\alpha)$ contains multiples of certain Milnor manifolds. Most such applications are a little difficult to state in terms of properties of $Y$ alone without the presence of additional structure. We will therefore make the following assumptions :

(1) $Y$ is $2 k-2$ connected;

(2) $\pi_{2 k-1}(Y) \cong Z_{p}, p$ a prime with generator $h: S^{2 k-1} \rightarrow Y$;

(3) $H_{*}(Y ; Z)$ is free abelian, $* \geq 2 k$.

Under these conditions we obtain:

Theorem 3.3. Under the above hypotheses we let a denote the generator of $H^{2 k-1}(Y ; Z) \cong Z_{p}$. Then

$$
\left[V^{2 p^{i-2}}\right]+d_{i} \in A(a)
$$

iff

$$
S_{i} \beta a \neq 0 \in H^{*}\left(Y ; Z_{p}\right) .
$$

Proof. Our hypotheses (1) and (2) readily imply that

(a) $X$ is $2 k-1$ connected, and

(b) $H_{*}(X ; Z)$ is free abelian. 
From the cofibration sequence

$$
Y \underset{g}{\longrightarrow} X \underset{f}{\longrightarrow} S^{2 k}
$$

we obtain the exact sequence

$$
\begin{gathered}
0 \longleftarrow H^{2 k}(Y ; Z) \stackrel{g^{*}}{\longleftarrow} H^{2 k}(X ; Z) \stackrel{f^{*}}{\longleftarrow} H^{2 k}\left(S^{2 k} ; Z\right) \\
Z * \oplus(\oplus Z) \oplus Z
\end{gathered}
$$

and thus we find $f^{*}(i)=p \cdot \hat{a}, \hat{a} \in H^{2 k}(X ; Z)$. From the properties of the Bockstein homomorphism we find

$$
g^{*}(\hat{a})=\beta(a) \in H^{2 k}\left(Y ; Z_{p}\right) .
$$

According to (2.4) and (3.1)

$$
\left[V^{2 p^{i-2}}\right]+d_{i} \in A(\alpha)
$$

iff

$$
S_{i} \hat{a} \neq 0 \in H^{*}\left(X ; Z_{p}\right)^{\prime \prime},
$$

where we have written $\hat{a}$ for its own $Z_{p}$ reduction. However $\operatorname{deg} S_{i}>1$ and

$$
g^{*}: H^{*}\left(X ; Z_{p}\right) \rightarrow H^{*}\left(Y ; Z_{p}\right)
$$

is an isomorphism for $*>2 k+1$ we have

$$
S_{i} \hat{a} \neq 0 \in H^{*}\left(X ; Z_{p}\right)
$$

iff

$$
g^{*}\left(S_{i}(\hat{a})\right)=S_{i} \beta(a) \neq 0 \in H^{*}\left(Y ; Z_{p}\right)
$$

as required. q.e.d.

As an application let us consider for $Y$ a 3-cell complex

$$
Y=S^{2 k-1} \cup_{p} S^{2 k} \cup_{\alpha} e^{1}: 1>s k, \quad p \text { an odd prime . }
$$

Then the hypotheses of (3.3) are satisfied and we find that

$$
\left[V^{2 p-2}\right] \in A(\alpha)
$$

iff

$$
S_{i} \beta a \neq 0 \in H^{*}\left(Y ; Z_{p}\right) .
$$


Now recall that $S_{1}=P_{p}^{1}$ and note that for $S_{1} \beta$ to be non-zero we must have $1=2 k+2 p-2$ and hence $P_{p}^{1} a=0 \in H^{*}\left(Y ; Z_{p}\right)$. Therefore

$$
\left[V^{2 p-2}\right] \in A(\alpha) \leftrightarrows Q_{1} a \neq 0 \in H^{*}\left(Y ; Z_{p}\right)
$$

where $Q_{1}=\left[\beta, P_{p}^{1}\right]$. Thus the attaching map

$$
\alpha: S^{2 p-2} \rightarrow S^{2 k-1} \cup_{p} S^{2 k}
$$

must be the coextension [19,pp. 13-15] of the element $\alpha_{1} \in \pi_{2 p-3}^{s}$ of Hopf invariant one $\bmod p$. The resulting complex $Y$ has been studied in some detail in [12], [13] where the same conclusions were reached on the basis of an Adams spectral sequence argument. As in [13] let us denote the resulting space by $V(1 / 2)$ (see [13] for a rationale of the notation). Thus

$$
V(1 / 2)=S^{2 k-1} \cup_{p} e^{2 k} \cup_{a_{1}} e^{2 k+2 p-2} .
$$

(Warning: the indexing is slightly different from [13] to conform with our discussion in $\S \S 1$ and 2.) As in [7], [12] let us pose the question of finding the spherical bordism classes on $V(1 / 2)$. In [13] we showed by a long involved argument that the only such classes are the ones you can see with your naked eye. A key step in the proof was to show $\left[V^{2 p^{i-1}}\right] \alpha$ could not be spherical for $i>1$. This now drops rather easily out of our previous work. For suppose to the contrary that $\left[V^{2 p^{i-2}}\right] \alpha$ is a spherical bordism class. Let

$$
h: S^{2 k-1+2 p^{i-2}} \rightarrow V(1 / 2)
$$

represent it and form the space

$$
Y=V(1 / 2) \cup_{h} e^{2 k+2 p i-2} .
$$

Then clearly

$$
V^{2 p{ }^{i-2}} \alpha=0 \in \tilde{\Omega}_{*}^{U}(Y)
$$

where by abuse of notation we have written $\alpha$ again for canonical generator of $\tilde{\Omega}_{2 k-1}(Y)$. Therefore according to (3.3) we must have

$$
S_{i} \beta a \neq 0 \in H^{*}\left(Y ; Z_{p}\right),
$$

where $a \in H^{2 k-1}\left(Y ; Z_{p}\right)$ is the canonical generator. Now note that

$$
H^{i}\left(Y ; Z_{p}\right) \cong \begin{cases}0, & i=0, \\ Z_{p}, & i=2 k-1,2 k, 2 k+2 p-2,2 k+2 p^{i}-2 . \\ 0, & \text { otherwise }\end{cases}
$$


For $i>2$ we have

$$
S_{i} \beta a=P_{p}^{p i-1} S_{i-1} \beta a-S_{i-1} P_{p}^{p i-1} a
$$

but

$$
\begin{aligned}
P_{p}^{p i-1} \beta a \in H^{2 k+2(p i-1)(p-1)}\left(Y ; Z_{p}\right) & =0, \\
S_{i-1} \beta a H^{2 k+2 p^{i-1-2}}\left(Y ; Z_{p}\right) & =0,
\end{aligned}
$$

and hence

$$
S_{i} \beta a=0 \in H^{*}\left(y ; Z_{p}\right),
$$

so these cases cannot occur. For $i=2$ we find

$$
S_{2} \beta a=P_{p}^{p} S_{1} \beta a-S_{1} P_{p}^{p} \beta a .
$$

Observe

$$
P_{p}^{p} \beta a \in H^{2 k+2 p(p-1)}\left(Y ; Z_{p}\right)=0,
$$

and hence

$$
S_{2} \beta a=P_{p}^{p} S_{1} \beta a
$$

But since

$$
H^{j}\left(Y ; Z_{p}\right)=0, \quad 2 k+2 p-2<j<2 k+2 p^{j}-2,
$$

it follows from [11] that

$$
P_{p}^{p}\left(S_{1} \beta a\right)=0
$$

Therefore

$$
S_{2} \beta a=0 \in H^{*}\left(Y ; Z_{p}\right)
$$

in this case also it too is ruled out.

While not as complete as the results of [13] the above computations do serve to indicate the utility of the preceeding results.

\section{Mapping spaces into spheres II}

In this section we wish to specialize somewhat our work of $\S 3$. For the sake of avoiding too many technicalities we will therefore make the following assumptions throughout the remainder of this section:

(1) $X$ is $2 k-1$ connected; 
(2) $H_{*}(X ; Z)$ is free abelian;

( 3 ) $f: X \rightarrow S^{2 k}$ is a continuous map;

(4) $H^{2 k}(X ; Z) \cong Z$ with generator $a$ and $f^{*} i=d a, d \in Z, d \neq 0$.

As before we form the cofibration sequence

$$
X \underset{f}{\longrightarrow} S^{2 k} \underset{h}{\longrightarrow} Y=S^{2 k} \cup_{f} c X .
$$

Note that $Y$ is also $2 k-1$ connected and $H_{j}(Y ; Z)$ is free abelian for $j \geq 2 k$. We again use the common symbol $\sigma$ to denote the generator of the groups

$$
\tilde{\Omega}_{2 k}^{f r}\left(S^{2 k}\right) \cong \tilde{\Omega}_{2 k}^{U}\left(S^{2 k}\right) \cong H_{2 k}\left(S^{2 k} ; Z\right) \cong Z
$$

we let

$$
h_{*}(\sigma)=\alpha \in \tilde{\Omega}_{2 k}^{f r}(Y) \cong \tilde{\Omega}_{2 k}^{U}(Y) \cong H_{2 k}(Y ; Z) \cong Z_{d} .
$$

Since $H_{*}(Y ; Z)$ is not free abelian we know [6, Cor. 3.11] that hom. $\operatorname{dim}_{\Omega_{*}^{U}} \tilde{\Omega}_{*}^{U}(Y) \geq 1$. We begin with the analog of (3.1)

Lemma 4.1. With the above notations the annihilator ideal $A(\alpha) \subset \Omega_{*}^{U}$ of $\alpha$ is isomorphic with the image of $f_{*}: \tilde{\Omega}_{*}^{U}(X) \rightarrow \tilde{\Omega}_{*}^{U}\left(S^{2 k}\right)$ by an $\Omega_{*}^{U}$-module isomorphism of degree $2 k$. Furthermore

$$
\text { hom. } \operatorname{dim}_{\Omega_{*}^{U}} \Omega_{*}^{U}(Y)=1+\text { hom. } \operatorname{dim}_{\Omega_{*}^{U}} A(\alpha) \text {. }
$$

Proof. The first assertion is clearly equivalent to (3.1). To obtain the second assertion we introduce the exact triangle

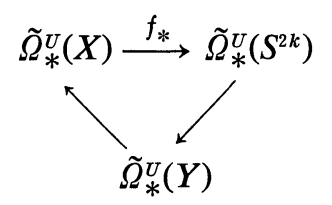

which yields the exact sequence

$$
0 \rightarrow \operatorname{coker} f_{*} \rightarrow \tilde{\Omega}_{*}^{U}(Y) \rightarrow \operatorname{ker} f_{*} \rightarrow 0
$$

of $\Omega_{*}^{U}$-modules. As $H_{*}(X ; Z)$ is free abelian it follows that $\tilde{\Omega}_{*}^{U}(X)$ is a free $\Omega_{*^{-}}^{U}$ module. The exact sequence

$$
0 \rightarrow \operatorname{ker} f_{*} \rightarrow \tilde{\Omega}_{*}^{U}(X) \rightarrow \operatorname{Im} f_{*} \rightarrow 0
$$

therefore shows

$$
1+\text { hom. } \operatorname{dim}_{\Omega_{*}^{U}} \operatorname{ker} f_{*} \geq \text { hom. } \operatorname{dim}_{\Omega_{*}^{U}} \operatorname{Im} f_{*}
$$

with equality holding except when the right hand side is 0 . From our first isomorphism and the exact sequence

$$
0 \rightarrow \operatorname{Im} f_{*} \rightarrow \tilde{\Omega}_{*}^{U}\left(S^{2 k}\right) \rightarrow \operatorname{coker} f_{*} \rightarrow 0
$$

we obtain 


$$
\text { hom. } \operatorname{dim}_{\Omega_{*}^{U}} \text { coker } f_{*}=1+\text { hom. } \operatorname{dim}_{\Omega_{*}^{U}} A(\alpha) \text {. }
$$

Applying [4, Chap. VI, Prop. 2.1] and elementary considerations to (A) yields in view of $(C)$ and $(E)$ that

$$
\text { hom. } \operatorname{dim}_{\Omega_{*}^{U}} \tilde{\Omega}_{*}^{U}(Y)=1+\text { hom. } \operatorname{dim}_{\Omega_{*}^{U}} A(\alpha)
$$

as desired.

Lemma 4.2. With the above notations we have:

(1) the cokernal of the Thom homomorphism

$$
\mu: \Omega_{2 k+m+1}^{U}(Y) \rightarrow H_{2 k+m+1}(Y ; Z)
$$

is isomorphic to

$$
\left[A(\alpha) \otimes_{\Omega_{*}^{U}} Z\right]_{m^{\prime}}
$$

$m>0$

(2) the kernel of the reduced Thom homomorphism

$$
\mu:\left[Z \otimes_{\Omega_{*}^{U}} \Omega_{*}^{U}(Y)\right]_{2 k+m+1} \rightarrow H_{2 k+m+1}(Y ; Z)
$$

is isomorphic to

$$
\operatorname{Tor}_{1, m}^{Q U}(Z, A(\alpha))
$$

$m>0$.

Proof. Recall that we have a cofibration

$$
S^{2 k} \stackrel{h}{\longrightarrow} Y \stackrel{g}{\longrightarrow} X .
$$

Thus we may regard $X$ as obtained from $Y$ by attaching a cell by the map $h$. In [6, Th. 11.3] we developed an exact sequence for such a situation. Recalling that $H_{*}(X ; Z)$ is free abelian yields the desired conclusion by inspection of [6, Th. 11. 3]. q.e.d.

We are now able to obtain one characterization of the condition hom. $\operatorname{dim}_{\Omega_{U}^{U}} \Omega_{*}^{U}(Y)=1$.

Proposition 4.3. With the above notations we have:

$$
\text { hom. } \operatorname{dim}_{\Omega_{*}^{U}} \Omega_{*}^{U}(Y)=1 \rightleftarrows A(\alpha)=d \Omega_{*}^{U} .
$$

Proof. The implication $\leftarrow$ is a consequence of (4.1) while $\rightarrow$ is [6, Prop. 5.9].

Notations. $\eta$ is the canonical line bundle over $S^{2 k}$ obtained via periodicity, so that $\eta-1 \in \tilde{K}\left(S^{2 k}\right) \cong Z$ is a generator.

From (2.1) and (2.2) we now obtain: 
Theorem 4.4. The value of hom. $\operatorname{dim}_{\Omega_{*}^{U}} \Omega_{*}^{U}(Y)$ is 1 if and only if the image $f !(\eta-1)$ in $K(X)$ is divisible by $d$.

Theorem 4.5. The value of hom. $\operatorname{dim}_{\Omega_{*}^{U}} \Omega_{*}^{U}(Y)$ is 1 if and only if $T d[W] \equiv 0$ $\bmod d$ for all $[W] \in A(\alpha)$.

We proceed now to a characterization of the condition hom. $\operatorname{dim}_{\Omega_{*}^{U}} \Omega_{*}^{U}(Y) \leq 2$. This will involve the relation of complex bordism to connective $k$-theory [6, $\S 10]$.

Theorem 4.6. The value of hom. $\operatorname{dim}_{\Omega_{*}^{U}} \Omega_{*}^{U}(Y)$ is at most 2 iff the reduced Thom homomorphism

$$
\tilde{\mu}: Z \otimes_{\Omega_{*}^{U}} \Omega_{*}^{U}(Y) \rightarrow H_{*}(Y ; Z)
$$

is a monomorphism.

We ask the reader to refer to $[6, \S 7]$. Also from $[13 ; \S 6]$ we find that there is a complex $V$ for which hom. $\operatorname{dim}_{\Omega_{*}^{U}} \Omega_{*}^{U}(V)>2$ but the reduced Thom map $\tilde{\mu}: Z \otimes_{\Omega_{*}} \Omega_{*}^{U}(V) \rightarrow H_{*}(V ; Z)$ is a monomorphism. In view of this we might regard (2.7) as quite unexpected.

There is a natural transformation $\Omega_{*}^{U}(\quad) \stackrel{\eta}{\longrightarrow} k_{*}(\quad)$, the homology theory based on the connective bu-spectrum. We recall that $k_{*}$ (point) $\cong Z[t]$, the integral polynomial ring on a single 2-dimensional generator. There is the graded $\Omega_{*}^{U}$-module structure on $Z[t]$ given by $\left[M^{2 n}\right] \rightarrow \mathrm{Td}\left[M^{2 n}\right] t^{n}$ and obtained by $\eta$ restricted to a point. There is also the natural transformation $\zeta: k_{*}(\quad) \rightarrow$ $H_{*}(; Z)$ and as established in $[15]$ an exact triangle

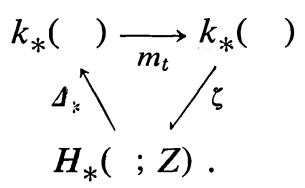

Notations. $I=\operatorname{ker}\left\{\mu: \Omega_{*}^{U} \rightarrow Z\right\}, I(t)=\operatorname{ker}\left\{\eta: \Omega_{*}^{U} \rightarrow Z[t]\right\}$.

Note that for any $\Omega_{*}^{U}$-module $M$,

$$
Z \otimes_{\Omega_{*}^{U}} M \cong M / I \cdot M, \quad Z[t] \otimes_{\Omega_{*}^{U}} M \cong M / I(t) \cdot M
$$

Recall that $I=([C P(1)], I(t))$ and hence

$$
I \cdot M=[C P(1)] \cdot M+I(t) \cdot M .
$$

Lemma 4.7. Let the notations be as before. Suppose that $y \in k_{*}(Y)$ with $A(y) \neq(0)$. Then $y=n t^{i} \eta(\sigma) n, i \in Z$.

Proof. Consider the exact sequence

$$
k_{*}\left(S^{2 k}\right) \underset{j_{*}}{\longrightarrow} k_{*}(Y) \underset{i_{*}}{\longrightarrow} k_{*}(X)
$$


Suppose that $y \in k_{*}(Y)$ and $A(y) \neq(0)$. Then $A(i * y) \neq(0)$. Since $k_{*}(\Sigma X)$ is a free $Z[t]$-module this implies $i * y=0$. Therefore $y=j * w$ for some $w \in k *\left(S^{2 k}\right)$. But since $j_{*}\left[S^{2 k}\right]=\eta(\sigma)$ generates the image of $j *$ as a $Z[t]$-module the result follows.

Proof of (4.6). Suppose that $\tilde{\mu}$ is monic. In view of [6, Th. 11.2] it will suffice to show that

$$
\tilde{\eta}: Z[t] \otimes_{\Omega_{*}^{U}} \Omega_{*}^{U}(Y) \rightarrow k_{*}(Y)
$$

is an isomorphism. This we do in two parts.

(1) $\tilde{\eta}$ is epic. Suppose that $y \in k_{*}(Y)$. According to [6, Th. 10.8] there exists an integer $i$ such that $t^{i} y \in \operatorname{Im}\left\{\eta: \Omega_{*}^{U}(Y) \rightarrow k_{*}(Y)\right\}$, As we wish to show $\eta$ epic we may as well assume $i>0$. Let

$$
t^{i} y=\eta x: x \in \Omega_{*}^{U}(Y) \text {. }
$$

Then by naturality,

$$
\mu x=\zeta \eta x=\zeta t^{i} y=0
$$

since $i>0$. Therefore

$$
x=[C P(1)] x^{\prime}+x^{\prime \prime}: x^{\prime \prime} \in I(t) \Omega_{*}^{U}(Y) .
$$

Thus

$$
\eta x=\eta\left([C P(1)] x^{\prime}+x^{\prime \prime}\right)=\operatorname{t\eta } x^{\prime}+\eta x^{\prime \prime}=t \eta x^{\prime \prime} .
$$

Therefore we have

$$
t^{i} y=\operatorname{t\eta } x^{\prime}
$$

and hence

$$
t\left(t^{i-1} y-\eta x^{\prime}\right)=0 .
$$

According to Lemma 2.8 this means

$$
t^{i-1} y-\eta x^{\prime}=n t^{j} \eta \sigma
$$

and hence

$$
t^{i-1} y=\eta\left(x^{\prime}+n[C P(1)]^{j} \sigma\right)
$$

Repeating the above argument shows that $y \in \operatorname{Im}$ and hence that $\tilde{\eta}$ is epic.

Before proving that $\tilde{\eta}$ is monic we require a lemma.

Lemma. The natural map $A(\sigma) \rightarrow A(\eta \sigma)$ is on to.

Proof. Recall that

$$
\text { coker }\left\{\mu: \Omega_{*}^{U}(Y) \rightarrow H_{*}(Y ; Z)\right\} \cong Z \otimes_{\Omega_{*}^{U}} A(\sigma)
$$

and for the same reason 


$$
\operatorname{coker}\left\{\eta: k_{*}(Y) \rightarrow H_{*}(Y ; Z)\right\} \cong Z \otimes_{Z[t]} A(\eta \sigma) .
$$

Hence the natural map $A(\sigma) \rightarrow A(\eta \sigma)$ induces an isomorphism

$$
Z \otimes_{Q_{*}^{U}} A(\sigma) \rightarrow Z[t] \otimes_{\Omega_{*}^{U}} A(\eta \sigma)
$$

As the natural map $A(\sigma) \rightarrow A(\eta \sigma)$ is $\Omega_{*}^{U} \rightarrow Z[t]$ sesquilinear the result follows. q.e.d.

We are now prepared to show $\tilde{\eta}$ is monic.

(2) $\tilde{\eta}$ is monic. Clearly it will suffice to show that $\operatorname{ker} \tilde{\eta}=I(t) \cdot \Omega_{*}^{U}(Y)$. We will proceed by induction over degree. As $\tilde{\eta}$ is monic in degree $2 k$ we proceed to the inductive step. Suppose that $x \in \Omega_{*}^{U}(Y)$ and $\eta x=0 \in k_{*}(Y)$. Then of course $\mu x=0 \in H_{*}(Y ; Z)$, and as we have assumed $\tilde{\mu}$ is monic this means $x \in I \cdot \Omega_{*}^{U}(Y)$ and hence

$$
x=[C P(1)] x^{\prime}+x^{\prime \prime}: x^{\prime \prime} \in I(t) \cdot \Omega_{*}^{U}(Y) .
$$

Now either $x^{\prime}=0$, in which case there is nothing to show, or

$$
0=\eta x=\operatorname{t\eta } x^{\prime}
$$

Hence, by (2.8),

$$
\eta x^{\prime}=n t^{i} \eta(\sigma)
$$

$n, i \in Z$, and hence $t \in A\left(n t^{i} \eta \sigma\right)$, i.e., $n t^{i+1} \in A(\eta \sigma)$. Hence there exists $[V] \in A(\sigma)$ with $\eta[V]=n t^{i+1}$. Thus we have

$$
\eta\left(n[C P(1)]^{i+1}-[V]\right)=0,
$$

and hence

$$
[W]=n[C P(1)]^{i+1}-[V] \in I(t) .
$$

But observe

$$
x^{\prime}-n[C P(1)]^{i} \sigma \in \operatorname{ker} \eta,
$$

and hence, by our inductive hypotheses,

$$
x^{\prime}-n[C P(1)]^{i} \sigma \in I(t) \cdot \Omega_{*}^{U}(Y) .
$$

Hence of course

$$
[C P(1)] x^{\prime}-n[C P(1)]^{i+1} \sigma \in I(t) \Omega_{*}^{U}(Y),
$$

and finally, since $[V] \in A(\sigma)$, 


$$
[C P(1)] x^{\prime}-n[C P(1)]^{i+1} \sigma=[C P(1)] x^{\prime}-n\left[C P(1)^{i+1}\right] \sigma+[V] \sigma,
$$

and $[W]=-n[C P(1)]^{i+1}+[V] \in I(t)$, we conclude

$$
[C P(1)] x^{\prime} \in I(t) \Omega_{*}^{U}(Y) \text {. }
$$

Therefore $x \in I(t) \cdot \Omega_{*}^{U}(Y)$ completing the inductive step.

Combining (1) and (2) we deduce that $\tilde{\eta}$ is an isomorphism and hence that hom. $\operatorname{dim}_{\Omega_{*}^{U}} \Omega_{*}^{U}(Y)$ is at most 2 .

The converse implication is contained in [6, Th. 4.4]. q.e.d.

To complete our discussion of the cases where the homological dimension of $\Omega_{*}^{U}(Y)$ is small we have

Corollary 4.8. Let the notations be as above. Then the homological dimension of $\Omega_{*}^{U}(Y)$ is at most 2 iff $\operatorname{Tor}_{1, *}^{\Omega_{*}^{U}}(Z, A(\sigma))=0$.

Proof. This follows immediately from (4.6), (4.1) and (4.2)(2).

Remark. Recently Dr. David Johnson has shown (University of Virginia Thesis, 1970) that for a finite complex $X$

$$
\eta: \Omega_{*}^{U}(X) \rightarrow k_{*}(X)
$$

is epic implies

$$
\tilde{\eta}: Z[t] \otimes_{\Omega_{*}^{U}} \Omega_{*}^{U}(X) \rightarrow k_{: *}(X)
$$

is an isomorphism. Thus the proof of (4.6) may be somewhat shortened by employing his result.

More recently Dr. Johnson and the second author of the present study have obtained a simplified proof of this result which is to appear shortly.

\section{Attaching cells II}

Let $Y$ be a finite complex, and $h: S^{2 k-1} \rightarrow Y$ a continuous map. As before we may attach a cell to $Y$ along $h$ to obtain $X=Y \cup_{h} e^{2 k}$. We will continue to study the interplay between $\tilde{\Omega}_{*}^{U}(Y), \tilde{\Omega}_{*}^{U}(X)$ and the annihilator ideal $A(\alpha) \subset \Omega_{*}^{U}$ of the spherical bordism element $\alpha=\left[S^{2 k-1}, h\right] \in \tilde{\Omega}_{*}^{U}(Y)$. We will place ourselves in a situation where the results of $\S 4$ may be applied to advantage. Let us therefore make the following assumptions

(1) $Y$ is $2 k-2$ connected,

(2) $\pi_{2 k-1}(Y)$ is finite cyclic with generator $[h]$,

(3) $H_{i}(Y ; Z)$ is free abelian for $i>2 k-1$.

Examples of such spaces abound. For example:

$1^{\circ}$. A Moore space $S^{2 k-1} \cup_{d} e^{2 k}$.

$2^{\circ}$. A space $V(1 / 2)=S^{2 k-1} \cup_{p} e^{2 k} \cup_{\phi} e^{2 k+2 p-2}$, where $p$ is an odd prime and $\phi: S^{2 k+2 p-3} \rightarrow S^{2 k-1} \cup_{p} e^{2 k}$ is as in $[13, \S 1]$. 
$3^{\circ}$. Let $\gamma: \Sigma^{2 k-1} C P(n) \rightarrow S^{2 k+1}$ be a map such that $\gamma^{*} i=d \Sigma^{2 k-1} c$, where $c \in H^{2}(C P(n) ; Z)$ is the canonical class. Let $Y=C(\gamma)$ be the mapping cone of $\gamma$.

The third example listed above is what motivated much of our work.

We obtain as previously a cofibration

$$
Y \underset{g}{\longrightarrow} X \underset{f}{\longrightarrow} S^{2 k}
$$

The following facts are now clear:

( 5 ) $X$ is $2 k-1$ connected;

(6) $H_{*}(X ; Z)$ is torsion free;

( 7 ) $f: X \rightarrow S^{2 k}$ is a continuous map;

( 8 ) $H^{2 k}(X ; Z) \cong Z$ with generator $a$ and $f^{*} i=d a, d \in Z, d \neq 0$, where $d$ is the order of $\pi_{2 k-1}(Y)$.

From (4.3) we thus obtain:

Proposition 5.1. With the above notations we have

$$
A(\alpha)=d \Omega_{*}^{U} \leftrightarrow \text { hom. } \operatorname{dim}_{\Omega_{*}^{U}} \Omega_{*}^{U}(Y)=1
$$

Proof. Immediate from (1)-(8) above and (4.3). q.e.d.

Note that (5.1) is really quite surprising as it says that if hom. $\operatorname{dim}_{\Omega_{*}^{U}} \Omega_{*}^{U}(Y)$ exceeds 1 , then it is possible to detect this fact simply from the study of $A(\alpha)$. Of course this "explains" why this was the case in the examples of the above type we have previously constructed. From (4.6) and (4.1) we now obtain:

Theorem 5.2. The value of hom. $\operatorname{dim}_{\Omega_{*}^{U}} A(\alpha)$ is at most 1 iff the reduced Thom homomorphism

$$
\tilde{\mu}: Z \otimes_{\Omega_{*}^{U}} \Omega_{*}^{U}(Y) \rightarrow H_{*}(Y ; Z)
$$

is a monomorphism.

Finally from (4.8) we obtain:

Corollary 5.3. With the above notations we have that the homological dimension of $A(\alpha)$ is at most 1 iff $\operatorname{Tor}_{1, *}^{\Omega U}(Z, A(\alpha))=0$.

This is strikingly similar to the condition [6, Cor. 3.11] that $\Omega_{*}^{U}(W)$ have projective dimension at most 1 , where $W$ is a finite complex. There is also the following closely related result which generalizes this.

Theorem 5.4. With the above notations we have that the following conditions are equivalent:

(1) hom. $\operatorname{dim}_{\Omega_{*}^{U}} \Omega_{*}^{U}(Y) \leq t+1$,

(2) hom. $\operatorname{dim}_{\Omega_{*}^{U}} A(\alpha) \leq t$,

(3) $\operatorname{Tor}_{j, *}^{O U}(Z, A(\alpha))=0$ for all $j \geq t$,

(4) $\operatorname{Tor}_{t * *}^{\Omega_{*}^{U}}(Z, A(\alpha))=0$,

for all positive integers $t$. 
Proof. The equivalence of (1) and (2) is a consequence of (4.1). For $t=1$ the result then follows from (5.3). However we will not rely on this as we have a simple argument for the general case.

As usual our basic tool is the cofibration

$$
Y \underset{g}{\longrightarrow} X \underset{f}{\longrightarrow} S^{2 k}
$$

and the associated $U$-bordism exact triangle

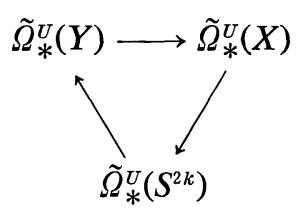

from which we obtain the exact sequence

$$
\begin{aligned}
& 0 \rightarrow \Sigma^{2 k} A(\alpha) \rightarrow \tilde{\Omega}_{*}^{U}\left(S^{2 k}\right) \rightarrow M(\alpha) \rightarrow 0, \\
& 0 \rightarrow M(\alpha) \underset{h_{*}}{\longrightarrow} \tilde{\Omega}_{*}^{U}(Y) \rightarrow C(\alpha) \rightarrow 0, \\
& 0 \rightarrow C(\alpha) \rightarrow \tilde{\Omega}_{*}^{U}(X) \underset{f_{*}}{\longrightarrow} \Sigma^{2 k} A(\alpha) \rightarrow 0,
\end{aligned}
$$

which define the $\Omega_{*}^{U}$-modules $M(\alpha)$ and $C(\alpha)$. As $\tilde{\Omega}_{*}^{U}\left(S^{2 k}\right)$ is a free $\Omega_{*}^{U}$-module the long exact sequence for $\operatorname{Tor}_{*, *}^{\Omega_{*}^{U}}(Z,-)$ applied to the top exact sequence gives an isomorphism

$$
\operatorname{Tor}_{j+1, *}^{\Omega_{*}^{U}}(Z, M(\alpha)) \stackrel{\cong}{\longrightarrow} \operatorname{Tor}_{j, *}^{\Omega_{*}^{U}}(Z, A(\alpha))
$$

for all $j \geq 0$.

Now supporse that hom. $\operatorname{dim}_{\Omega_{*}^{U}} \Omega_{*}^{U}(Y) \leq t$. Then according to [6, Cor. 3.11] we have

$$
\operatorname{Tor}_{i, *}^{\Omega_{*}^{U}}\left(Z, \Omega_{*}^{U}(Y)\right)=0 \quad \text { for all } i \geq t+1 .
$$

Let now $j \geq t$. Then the middle exact sequence above provides an isomorphism

$$
\operatorname{Tor}_{j+2, *}^{\Omega_{U}^{U}}(Z, C(\alpha)) \stackrel{\cong}{\longrightarrow} \operatorname{Tor}_{j+1, *}^{\Omega^{U}}(Z, M(\alpha))
$$

Finally we note that condition (6) above and [6, Prop. 3.3] imply that $\widetilde{\Omega}_{*}^{U}(X)$ is a free $\Omega_{*}^{U}$-module. Therefore the last exact sequence above provides in a similar way an isomorphism

$$
\operatorname{Tor}_{j+3, *}^{\Omega_{*}^{U}}(Z, A(\alpha)) \stackrel{\cong}{\longrightarrow} \operatorname{Tor}_{j+2, *}^{\Omega_{*}^{U}}(Z, C(\alpha))
$$


Thus, to summarize, we have for any integer $j \geq t-1$ an isomorphism

$$
\operatorname{Tor}_{j, *}^{\Omega_{*}^{U}}(Z, A(\alpha)) \cong \operatorname{Tor}_{j+3, *}^{\Omega_{*}^{U}}(Z, A(\alpha))
$$

and hence an isomorphism

$$
\operatorname{Tor}_{j, *}^{\Omega_{*}^{U}}(Z, A(\alpha)) \cong \operatorname{Tor}_{j+3 s, *}^{\Omega U}(Z, A(\alpha))
$$

for any nonnegative integer $s$. But recall that $A(\alpha)$ has finite projective dimension as an $\Omega_{*}^{U}$-module [6, Prop. 5.1] and hence

$$
\operatorname{Tor}_{j+3 s, *}^{\Omega U}(A, A(\alpha))=0
$$

for $s$ large. Therefore

$$
\operatorname{Tor}_{j, *}^{\Omega_{*}^{U}}(Z, A(\alpha))=0
$$

as desired, showing (1) $\rightarrow$ (3).

To obtain the reverse implication note $(3) \rightarrow(4)$ trivially, and we reason as follows. From the first and last sequences above we have isomorphisms

$$
\begin{aligned}
& \operatorname{Tor}_{r+1, *}^{\Omega_{U}^{U}}(Z, M(\alpha)) \cong \operatorname{Tor}_{r, *}^{\Omega_{U}^{U}}(Z, A(\alpha)), \\
& \operatorname{Tor}_{s, *}^{\Omega_{*}^{U}}(Z, C(\alpha)) \cong \operatorname{Tor}_{s+1, *}^{\Omega_{*}^{U}}(Z, A(\alpha)) .
\end{aligned}
$$

for all $r, s>0$. Therefore the long exact sequence derived from the middle exact sequence

$$
\cdots \rightarrow \operatorname{Tor}_{j, *}^{\Omega^{U}}(Z, M(\alpha)) \rightarrow \operatorname{Tor}_{j, *}^{\Omega_{*}^{U}}\left(Z, \tilde{\Omega}_{*}^{U}(Y)\right) \rightarrow \operatorname{Tor}_{j, *}^{\Omega^{U}}(Z, C(\alpha)) \rightarrow
$$

may be written

$$
\operatorname{Tor}_{j-1, *}^{\Omega_{W}^{U}}(Z, A(\alpha)) \rightarrow \operatorname{Tor}_{j, *}^{\Omega U}\left(Z, \tilde{\Omega}_{*}^{U}(Y)\right) \rightarrow \operatorname{Tor}_{j+1, *}^{\Omega_{*}^{U}}(Z, A(\alpha)) \rightarrow .
$$

Put now $j=t+1$ above and we find

$$
0 \rightarrow \operatorname{Tor}_{t+1, *}^{\Omega_{U}^{U}}\left(Z, \tilde{\Omega}_{*}^{U}(Y)\right) \rightarrow 0
$$

and hence

$$
\operatorname{Tor}_{t+1, *}^{\Omega_{U}^{U}}\left(Z, \tilde{\Omega}_{*}^{U}(Y)\right)=0 .
$$

Therefore according to $\left[6\right.$, Cor. 3.11] hom. $\operatorname{dim}_{\Omega_{*}^{U}} \tilde{\Omega}_{*}^{U}(Y) \leq t+1$ as required. Thus (4) $\rightarrow(1)$ and the theorem is established.

Thus for the special complexes $Y$ the homological dimension of $\tilde{\Omega}_{*}^{U}(Y)$ may be completely determined from studying properties of $A(\alpha)$ akin to ones we have already investigated for spaces. 


\section{A relation to connective $k$-theory}

In the previous two section we have studied in some detail the homological dimension of the complex bordism module of a restricted class of spaces, namely, those spaces $Y$ such that

(1) $Y$ is $2 k-2$ connected,

(2) $\pi_{2 k-1}(Y)$ is finite cyclic,

(3) $H_{i}(Y ; Z)$ is free abelian for $i>2 k-1$.

We have found in (4.6) and (5.4) a satisfactory criteria for hom. $\operatorname{dim}_{\Omega_{*}} \Omega_{*}^{U}(Y)$ $=2$. Just as these conditions are related (4.6), (5.2) to the monomorphy of the Thom map

$$
\tilde{\eta}: Z \otimes_{\Omega_{*}^{U}} \Omega_{*}^{U}(Y) \rightarrow H^{*}(Y ; Z),
$$

so is the condition hom. $\operatorname{dim}_{\Omega_{*}^{U}} \Omega_{*}^{U}(Y)=3$ related to the monomorphy of the reduced Thom map

$$
\tilde{\eta}: Z[t] \otimes_{\Omega_{*}^{U}} \Omega_{*}^{U}(Y) \rightarrow k_{*}(Y) .
$$

The demonstration of this fact will require some preliminary results on annihilator ideals of spherical bordism classes.

We therefore suppose given a finite complex $A$ and a spherical bordism class $\gamma \in \Omega_{n}^{U}(A)$. Represent $\gamma$ by a map $g: S^{n} \rightarrow A$ by passing to a suitable suspension of $A$ if necessary. Thus $\left[S^{n}, g\right]=\gamma \in \Omega_{n}^{U}(A)$.

Theorem 6.1. With the preceeding notations we have for each positive integer $m$ that

$$
m_{t}: \operatorname{Tor}_{m, *}^{\Omega_{*}^{U}}(Z[t], A(\gamma)) \unlhd
$$

is nilpotent, where $m_{t}$ denotes multiplication by $t \in Z[t]$.

Proof. We refer the reader to the second proof of [6, Prop. 10.4] for a detailed discussion of a very similar situation. As noted there it will clearly suffice to show that

$$
\operatorname{Tor}_{m, *}^{\delta_{*}^{U}}\left(Z\left[t, t^{-1}\right], A(\gamma)\right)=0, \quad m>0 .
$$

As usual we introduce the cofibration

$$
S^{n} \underset{g}{\longrightarrow} A \underset{\Psi}{\longrightarrow} B=A \cup_{g} e^{n+1},
$$

from which we obtain the exact sequences:

$$
\begin{aligned}
& 0 \rightarrow \Sigma^{n} A(\gamma) \rightarrow \tilde{\Omega}_{*}^{U}\left(S^{n}\right) \rightarrow M(\gamma) \rightarrow 0, \\
& 0 \rightarrow M(\gamma) \rightarrow \tilde{\Omega}_{*}^{U}(A) \rightarrow C(\gamma) \rightarrow 0, \\
& 0 \rightarrow C(\gamma) \rightarrow \tilde{\Omega}_{*}^{U}(B) \rightarrow \Sigma^{n} A(\gamma) \rightarrow 0 .
\end{aligned}
$$


Recall that the $Z$ graded version of the relation between $\Omega_{*}^{U}(\quad)$ and $K_{*}(\quad)$ is equivalent to [6, Prop. 10.4]

$$
\operatorname{Tor}_{m, *}^{\Omega_{*}^{U}}\left(Z\left[t, t^{-1}\right], \Omega_{*}^{U}(C)\right)=0, \quad m>0
$$

for any finite complex $C$. From the bottom exact sequence preceeding and the long exact sequence for Tor $(-,-)$ we therefore learn that for $m>0$

$$
\operatorname{Tor}_{m, *}^{\Omega_{*}^{U}}\left(Z\left[t, t^{-1}\right], \Sigma^{n} A(\gamma)\right) \cong \operatorname{Tor}_{m+1, *}^{\Omega_{*}^{U}}\left(Z\left[t, t^{-1}\right], C(\gamma)\right) .
$$

A similar argument applied to the middle exact sequence gives

$$
\operatorname{Tor}_{m+1, *}^{\Omega_{*}^{U}}\left(Z\left[t, t^{-1}\right], C(\gamma)\right) \cong \operatorname{Tor}_{m+2, *}^{\Omega_{*}^{U}}\left(Z\left[t, t^{-1}\right], M(\gamma)\right),
$$

and finally appealing to the top exact sequence we find

$$
\operatorname{Tor}_{m+2, *}^{\Omega_{*}^{U}}\left(Z\left[t, t^{-1}\right], M(\gamma)\right) \cong \operatorname{Tor}_{m+3, *}^{\Omega_{U}^{U}}\left(Z\left[t, t^{-1}\right], \Sigma^{n} A(\gamma)\right) .
$$

Stringing these isomorphisms together then gives

$$
\operatorname{Tor}_{m, *}^{\Omega_{*}^{U}}\left(Z\left[t, t^{-1}\right], \Sigma^{n} A(\gamma)\right) \cong \operatorname{Tor}_{m+3, *}^{\Omega_{*}^{U}}\left(Z\left[t, t^{-1}\right], \Sigma^{n} A(\gamma)\right)
$$

for any $m>0$, and hence for any $m>0$

$$
\operatorname{Tor}_{m, *}^{\Omega_{*}^{U}}\left(Z\left[t, t^{-1}\right], \Sigma^{n} A(\gamma)\right) \cong \operatorname{Tor}_{m+3 j, *}^{\Omega_{*}^{U}}\left(Z\left[t, t^{-1}\right], \Sigma^{n} A(\gamma)\right)
$$

for any $j \geq 0$. But by [6, Prop. 5.1] $\Sigma^{n} A(\gamma)$ has finite projective dimension and hence

$$
\operatorname{Tor}_{m+3 j *}^{\Omega U}\left(Z\left[t, t^{-1}\right], \Sigma^{n} A(\gamma)\right)=0
$$

for large $j$, which yields the result.

Corollary 6.2. With the preceeding notations we have that the conditions

(1) $\operatorname{Tor}_{m, *}^{\Omega_{*}^{U}}(Z[t], A(\gamma))=0, m \geq q$,

(2) $\operatorname{Tor}_{m, *}^{\Omega_{u}^{*}}(Z, A(\gamma))=0, m \geq q+1$, are equivalent for any $q \geq 0$.

Proof. Suppose that the second condition holds. Introduce the exact sequence of $\Omega_{*}^{U}$-modules

$$
0 \rightarrow Z[t] \underset{m_{t}}{\longrightarrow} Z[t] \rightarrow Z \rightarrow 0 .
$$

Applying the functor $\operatorname{Tor}_{*, *}^{\Omega_{*}^{U}}(-, A(\gamma))$ then leads to the long exact sequence

$$
\begin{aligned}
\cdots \longrightarrow \operatorname{Tor}_{r+1, *}^{\Omega_{*}^{U}}(Z, A(\gamma)) & \stackrel{\partial}{\longrightarrow} \operatorname{Tor}_{r, *}^{\Omega_{*}^{U}}(Z[t], A(\gamma)) \stackrel{m_{t}}{\longrightarrow} \operatorname{Tor}_{r, *}^{\Omega_{*}^{U}}(Z[t], A(\gamma)) \\
& \longrightarrow \operatorname{Tor}_{r, *}^{\Omega_{*}^{U}}(Z, A(\gamma)) \longrightarrow \cdots
\end{aligned}
$$

from which we obtain 


$$
m_{t}: \operatorname{Tor}_{r, *}^{\Omega_{*}^{U}}(Z[t] A(\gamma)) \unlhd \begin{cases}\text { is iso for } & r \geq m+1 \\ \text { is monic for } & r=m\end{cases}
$$

In view of (6.1) this yields (1). The reverse implication follows by reversing the steps in the above argument.

We now arrive at the promised result relating the condition hom. $\operatorname{dim}_{\Omega_{*}^{U}} \Omega_{*}^{U}(Y)$ $=3$ to the monomorphy of

$$
\tilde{\eta}: Z[t] \otimes_{\Omega_{*}^{U}} \Omega_{*}^{U}(Y) \rightarrow k_{*}(Y) .
$$

We therefore revert to the situation described at the beginning of $\S 5$ with $h: S^{2 k-1} \rightarrow Y$ a map representing a generator of the finite cyclic group $\pi_{2 k-1}(Y)$ of the $2 k-2$ connected finite complex $Y$ whose integral homology groups are free abelian in dimensions greater than $2 k-1$.

Theorem 6.3. The value of hom. $\operatorname{dim}_{\Omega_{*}^{U}} \Omega_{*}^{U}(Y)$ is at most 3 if and if the reduced Thom homomorphism

$$
\tilde{\eta}: Z[t] \otimes_{\Omega_{*}^{U}} \Omega_{*}^{U}(Y) \rightarrow k_{*}(Y)
$$

is monic.

Proof. Suppose that hom. $\operatorname{dim}_{\Omega_{*}^{U}} \Omega_{*}^{U}(Y) \leq 3$. Then according to (4.1) hom. $\operatorname{dim}_{\Omega_{*}^{U}} A(\alpha) \leq 2$ and hence by (5.4)

$$
\operatorname{Tor}_{j, *}^{\Omega_{*}^{U}}(Z, A(\alpha))=0, \quad j \geq 2 .
$$

Therefore by (6.2) we obtain

$$
\operatorname{Tor}_{j, *}^{\Omega_{*}^{U}}(Z[t], A(\alpha))=0, \quad j \geq 1,
$$

and the result follows from the $k_{*}$-theoretic analog of [6, Th. 11.3](see the remarks preceding [6, Th. 11.4]).

Conversely, if

$$
\tilde{\eta}: Z[t] \otimes_{\Omega_{*}^{U}} \Omega_{*}^{U}(Y) \rightarrow k_{*}(Y)
$$

is monic, then as just noted

$$
\operatorname{Tor}_{1, *}^{\Omega U}(Z[t], A(\alpha))=0 .
$$

From the cofibration

$$
S^{2 k-1} \stackrel{h}{\longrightarrow} Y \underset{g}{\longrightarrow} X
$$

We obtain as usual 


$$
\begin{aligned}
& 0 \rightarrow \Sigma^{2 k-1} A(\alpha) \rightarrow \Omega_{*}^{U}\left(S^{2 k-1}\right) \rightarrow M(\alpha) \rightarrow 0, \\
& 0 \rightarrow M(\alpha) \rightarrow \Omega_{*}^{U}(Y) \rightarrow C(\alpha) \rightarrow 0, \\
& 0 \rightarrow C(\alpha) \rightarrow \Omega_{*}^{U}(X) \rightarrow \Sigma^{2 k-1} A(\alpha) \rightarrow 0
\end{aligned}
$$

The first and last of these sequences yield isomorphisms

$$
\begin{aligned}
& \operatorname{Tor}_{r+1, *}^{\Omega_{*}^{U}}(Z[t], M(\alpha)) \cong \operatorname{Tor}_{r, *}^{\Omega_{*}^{U}}\left(Z[t], \Sigma^{2 k-1} A(\alpha)\right), \\
& \operatorname{Tor}_{s, *}^{\Omega_{*}^{U}}(Z[t], C(\alpha)) \cong \operatorname{Tor}_{s+1, *}^{\Omega_{*}^{U}}\left(Z[t], \Sigma^{2 k+1} A(\alpha)\right),
\end{aligned}
$$

and thus the middle exact sequence gives

$$
0=\operatorname{Tor}_{2, *}^{\Omega_{*}^{U}}(Z[t], M(\alpha)) \rightarrow \operatorname{Tor}_{2, *}^{\Omega_{*}^{U}}\left(Z[t], \Omega_{*}^{U}(Y)\right) \rightarrow \operatorname{Tor}_{2, *}^{\Omega_{*}^{U}}(Z[t], C(\alpha))=0,
$$

and hence

$$
\operatorname{Tor}_{2, *}^{Q_{*}^{U}}\left(Z[t], \Omega_{*}^{U}(Y)\right)=0,
$$

and the result follows from [6, Prop. 10.5].

Finally by analogy with (5.4) we obtain the following result whose proof is left the reader.

Theorem. 6.4. With the above notations we have that the following conditions are equivalent:

(1) hom. $\operatorname{dim}_{\Omega_{*}^{U}} \Omega_{*}^{U}(Y) \leq t+2$,

(2) hom. $\operatorname{dim}_{\Omega_{*}^{U}} A(\alpha) \leq t+1$,

(3) $\operatorname{Tor}_{j *}^{O_{*}^{*}}(Z[t], A(\alpha))=0$ for all $j \geq t$,

(4) $\operatorname{Tor}_{t, *}^{\Omega_{u}^{U}}(Z[t], A(\alpha))=0$,

for all positive integers $t$.

While it may seem surprising that a complex of the form $Y$ can have a hom. $\operatorname{dim}_{\Omega_{*}^{U}} \Omega_{*}^{U}(Y)=3$, this is indeed the case as is shown in Examples 1 and 2 of $\S 7$.

\section{A relation to $e_{C}$, and the Todd character}

To illustrate the relation of hom. $\operatorname{dim}_{\Omega_{*}^{U}} \Omega_{*}^{U}(\quad)$ to more familiar invariants of algebraic topology let us consider the Adams [2]-Toda [20] invariant $e_{C}$.

Suppose that $\gamma \in \pi^{s}{ }_{2 n-1}$ is an element of the stable $2 n-1$ stem with $m \gamma=0$. Represent $\gamma$ by a map

$$
g: S^{2 k+2 n-1} \rightarrow S^{2 k}
$$

Let

$$
m: S^{2 k} \rightarrow S^{2 k}
$$

represent the map of degree $m$. Then the composite 


$$
S^{2 k+2 n-1} \underset{g}{\longrightarrow} S^{2 k} \underset{m}{\longrightarrow} S^{2 k}
$$

is null homotopic and so we may extend $m$ to a map

$$
f: S^{2 k} \cup_{g} e^{2 k+2 n} \rightarrow S^{2 k}
$$

Let us write $Y$ for $S^{2 k} \cup_{g} e^{2 k+2 n}$ and put $X=C(f)=S^{2 k} \cup_{f} c Y$. There is then the cofibration

$$
Y \underset{f}{\longrightarrow} S^{2 k} \underset{h}{\longrightarrow} X
$$

We see that $X$ is a 3-cell complex and

$$
\widehat{H}_{2 k}(X ; Z)=Z_{m} ; \widehat{H}_{2 k+2 n+1}(X ; Z)=Z ; \hat{H}_{i}(X ; Z)=0 \text {, otherwise . }
$$

If $\alpha \in \widetilde{\Omega}_{2 k}(X) \cong Z_{m} \cong \widehat{H}_{2 k}(X ; Z)$ is the generator, then $A(\alpha)$ may be identified with the image of $f_{*}: \widetilde{\Omega}_{*}^{U}(Y) \rightarrow \tilde{\Omega}_{*}^{U}\left(S^{2 k}\right)$ by an isomorphism of degree $2 k$ (Lemma 4.1).

Now $\tilde{\Omega}_{*}^{U}(Y)$ has two generators, and one may be selected to be the inclusion $i: S^{2 k} \rightarrow Y$ while the other $\left[M^{2(n+k)}, \phi\right] \in \widetilde{\Omega}_{2 n+2 k}^{U}(Y)$ is characterized by the requirement that

$$
\mu\left[M^{2 n+2 k}, \phi\right] \in \widehat{H}_{2 n+2 k}(Y ; Z) \cong Z
$$

be the generator ${ }^{1}$. Denote these generators by $\gamma_{2 k} \in \Omega_{2 k}^{U}(Y), \gamma_{2 k+2 n} \in \Omega_{2 n+2 k}^{U}(Y)$ (note some choice had to be made for the second one). If $\gamma^{\prime}{ }_{2 k+2 n}$ is any other choice of a generator for $\Omega_{2 n+2 k}^{U}(Y)$, then

$$
\mu \gamma_{2 k+2 n}-\mu \gamma^{\prime}{ }_{2 k+2 n}=0
$$

Since $H_{*}(Y ; Z)$ is free abelian, the reduced Thom map

$$
\tilde{\mu}: Z \otimes_{\Omega_{*}^{U}} \Omega_{*}^{U}(Y) \rightarrow H_{*}(Y ; Z)
$$

is a monomorphism [6, Lemma 3.1]. Therefore $\gamma_{2 k+2 n}-\gamma_{2 k+2 n}^{\prime}$ must be decomposable, i.e.,

$$
\gamma_{2 k+2 n}-\gamma^{\prime}{ }_{2 k+2 n}=\left[W^{2 n}\right] \gamma_{2 k}
$$

for some $\left[W^{2 n}\right] \in \Omega_{2 n}^{U}$.

Returning now to our cofibration we note that

$$
f_{*} \gamma_{2 k}=m \sigma_{2 k}: \tilde{\Omega}_{2 k}^{U}\left(S^{2 k}\right) \cong Z \cdot \sigma_{2 k},
$$

and that

\footnotetext{
1 We assume that a choice of orientation has been made for the cells of $Y$, which imposes one on $X$, so we may speak of "the" generator.
} 


$$
f_{*} \gamma_{2 k+2 n}=\left[V^{2 n}\right] \sigma_{2 k}, \quad f_{*} \gamma^{\prime}{ }_{2 k+2 n}=\left[V^{\prime 2 n}\right] \sigma_{2 k}
$$

for unique elements $\left[V^{2 n}\right],\left[V^{\prime 2 n}\right] \in A(\alpha)$. Our previous discussion now shows that

$$
\begin{aligned}
f_{* \gamma_{2 k+2 n}-f_{*} \gamma_{2 k+2 n}^{\prime}} & =\left(\left[V^{2 n}\right]-\left[V^{\prime 2 n}\right]\right) \sigma_{2 k} \\
f_{*}\left[W^{2 n}\right] \gamma_{2 k} & =m\left[W^{2 n}\right] \sigma_{2 k}
\end{aligned}
$$

and hence

$$
\left[V^{2 n}\right]-\left[V^{\prime 2 n}\right] \in(m)
$$

Thus the class

$$
\left[V^{2 n}\right] \in \Omega_{*}^{U} /(m)
$$

is independent of the choice of generator for $\Omega_{2 k+2 n}^{U}(Y)$ and is a cobordism $e$ invariant of $\gamma \in \pi^{s}{ }_{2 n-1}$. More precisely we have:

Theorem 7.1. With the notations above we have

$$
e_{C}(\gamma)=-\frac{\operatorname{Td}\left[V^{2 n}\right]}{m} \in Q / Z
$$

Proof. Let

$$
a e H^{2 k}(Y ; Z) \cong Z, \quad b e H^{2 k+2 n}(Y ; Z) \cong Z
$$

be generators for the indicated cohomology groups. There is an $\xi \in \vec{K}(Y)$ with

$$
\operatorname{ch}(\xi)=a+e_{C}(g) b .
$$

We also have

$$
\operatorname{ch}\left(f^{*}(\eta-1)\right)=m a,
$$

where $\eta \in \hat{K}\left(S^{2 k}\right)$ is the canonical class. Let

$$
\gamma_{2 n+2 k}=\left[M^{2 n+2 k}, \phi\right] \in \Omega_{2 n+2 k}^{U}(Y)
$$

be a generator. Then by the Riemann Roch theorem [3]

$$
\left\langle\operatorname{ch} \phi^{!}(\xi) \mathrm{Td}^{-1}[M],[M]\right\rangle
$$

is a $K$-theory characteristic number of $[M$,$] and hence is an integer. By natu-$ rality of $c h$

$$
\operatorname{ch} \phi^{\prime} \xi=\phi^{*} \operatorname{ch} \xi=\phi^{*} a+e_{C}(g) \phi^{*}(b)
$$


Therefore

$$
\left\langle\phi^{*}(a) \operatorname{Td}^{-1}[M],[M]\right\rangle+\left\langle e_{C}(g) \phi^{*}(b) \operatorname{Td}^{-1}[M],[M]\right\rangle \in Z .
$$

Now observe

$$
\operatorname{deg} b=2 n+2 k=\operatorname{dim} M .
$$

Thus

$$
\left\langle e_{C}(g) \phi^{*}(b) \operatorname{Td}^{-1}[M],[M]\right\rangle=e_{C}(g)\left\langle\phi^{*}(b),[M]\right\rangle
$$

and by choice of $[M, \phi]$

$$
\left\langle\phi^{*}(b),[M]\right\rangle=1 .
$$

Thus

$$
\left\langle\phi^{*}(a) \operatorname{Td}^{-1}[M],[M]\right\rangle=-e_{C}(g) \in Q / Z .
$$

But according to our discussion of characteristic numbers preceding (2.1) we have

$$
\operatorname{Td}\left[V^{2 n}\right]=m\left\langle\phi^{*}(a) \operatorname{Td}^{-1}[M],[M]\right\rangle
$$

and therefore

$$
\frac{\operatorname{Td}\left[V^{2 n}\right]}{m}=\left\langle\phi^{*}(a) \operatorname{Td}^{-1}[M],[M]\right\rangle=-e_{C}(g) \in Q / Z,
$$

which is the desired conclusion.

Theorem 7.2. With the notations preceding we have that hom. $\operatorname{dim}_{\Omega_{*}^{U}} \Omega_{*}^{U}(X)$ $=2$ iff $e_{c}(g) \neq 0$.

Proof. First note that

$$
A(\alpha)=\left(m,\left[V^{2 n}\right]\right) .
$$

Thus according to (2.2) and (4.3) we learn that the homological dimension of $\Omega_{*}^{U}(X)$ is 1 iff $\operatorname{Td}\left[V^{2 n}\right] \equiv 0 \bmod m$. From (6.1) we have equivalence

$$
\operatorname{Td}\left[V^{2 n}\right] \equiv 0 \bmod m \rightleftarrows e_{C}(g)=0 \in Q / Z \text {. }
$$

Therefore we learn that

$$
e_{C}(g)=0 \rightarrow \text { hom. } \operatorname{dim}_{\Omega_{*}^{U}} \Omega_{*}^{U}(X)=1,
$$

and

$$
e_{C}(g) \neq 0 \rightarrow \text { hom. } \operatorname{dim}_{\Omega_{*}^{U}} \Omega_{*}^{U}(X)>1
$$


It therefore remains to show that the homological dimension of $\Omega_{*}^{U}(X)$ cannot exceed 2. To observe that $X$ has a cell structure

$$
X=S^{2 k} \cup_{m} e^{2 k+1} \cup_{g} e^{2 k+2 n+1},
$$

that is, the space $X$ may be made as follows. Consider

$$
S^{2 k+2 n-1} \underset{g}{\longrightarrow} S^{2 k} \underset{m}{\longrightarrow} S^{2 k}
$$

Since this composition is null homotopic we may choose a co-extension [19, p. 13]

$$
G: S^{2 k+2 n} \rightarrow S^{2 k} \bigcup_{m} e^{2 k+1}
$$

of $g$. The space $X$ is then the same homotopy type as the mapping cone of $G$, i.e.,

$$
X=S^{2 k} \cup_{m} e^{2 k+1} \cup_{G} e^{2 k+2 n+1} .
$$

Now clearly the homological dimension of $\Omega_{*}^{U}\left(S^{2 k} \cup_{m} e^{2 k+1}\right)$ is exactly 1 . Therefore according to [6, Th. 5.4] the homological dimensian of $\Omega_{*}^{U}(X)$ cannot exceed 2 . Combining this with our previous discussion we obtain the equivalences

$$
e_{C}(g)=0 \rightleftarrows \text { hom. } \operatorname{dim}_{\Omega_{*}^{U}} \Omega_{*}^{U}(X)=1,
$$

and

$$
g_{C}(g) \neq 0 \rightleftarrows \text { hom. } \operatorname{dim}_{\Omega_{*}^{U}} \Omega_{*}^{U}(X)=2
$$

as desired.

The preceding discussions of the invariant $e_{C}$ and the Todd genus can be put more in the style of $[20],[2, \S 7]$ by introducing a natural transformation

$$
\text { th: } \Omega_{*}^{U}(\quad) \rightarrow H_{*}(; Q)
$$

of $Z_{2}$-graded homology theories which we will call the Todd character. Its definition depends on the duality between

$$
H_{*}(A ; Q) \text { and } \operatorname{Hom}\left(H^{*}(A ; Q), Q\right)
$$

whenever $A$ is a finite complex, and runs as follows. Let $A$ be a finite complex and identify $H_{*}(A ; Q)$ with $\operatorname{Hom}\left(H^{*}(A ; Q), Q\right)$ by the natural map

$$
\rho: H_{*}(A ; Q) \rightarrow \operatorname{Hom}\left(H^{*}(A ; Q), Q\right)
$$

given by 


$$
\rho(x)(y)=\langle y, x\rangle: y \in H^{*}(A ; Q), x \in H_{*}(A ; Q) .
$$

Define

$$
\text { th: } \Omega_{*}^{U}(A) \rightarrow H_{*}(A ; Q)
$$

by

$$
\rho(\operatorname{th}[M, \phi])(y)=\left\langle\phi^{*}(y) \mathrm{Td}^{-1}[M],[M]\right\rangle \in Q .
$$

(Note that if $y=\operatorname{ch}(\xi)$ for some $\xi \in K(A)$, then the right hand side will actually be an integer by the $R R$ theorem.)

The relation between the Todd character and the invariant $e_{C}$ may be seen to be as follows. Suppose that $\gamma \in \pi^{s}{ }_{2 n-1}$. Represent $\gamma$ by a map

$$
g: S^{2 n+2 k-1} \rightarrow S^{2 k}
$$

and form the cofibration

$$
S^{2 k} \underset{f}{\longrightarrow} A \underset{h}{\longrightarrow} S^{2 n+2 k}: A=S^{2 k} \cup_{g} e^{2 n+2 k}
$$

Then $\Omega_{*}^{U}(A)$ is a free $\Omega_{*}^{U}$-module with two generators, one of which, $\gamma_{2 k}$, may be chosen to be $\left[S^{2 k}, f\right]$. The other, $\gamma_{2 n+2 k}$, may be taken to be any class with $h_{*} \gamma_{2 n+2 k}=\sigma_{2 n+2 k}$, where $\sigma_{2 n+2 k} \in H_{2 n+2 k}\left(S^{2 n+2 k}\right)$ is the canonical generator. Let $a \in H_{2 n}(A ; Z)$ and $b \in H_{2 n+2 k}(A ; Z)$ be corresponding homology generators. Then

$$
\operatorname{th}\left(\gamma_{2 n+2 k}\right)=b+\lambda a, \quad \lambda \in Q .
$$

The generator $\gamma_{2 n+2 k}$ is not unique of course, but may be altered by adding a factor of the form $\left[M^{2 k}\right] \gamma_{2 n}$. Thus $\lambda \in Q$ is not an invariant of $\gamma$. But observe

$$
\operatorname{th}\left[M^{2 k}\right] \gamma_{2 n}=\operatorname{Td}\left[M^{2 k}\right] a
$$

Since $\operatorname{Td}\left[M^{2 k}\right] \in Z$, the residue class $\lambda \in Q / Z$ becomes well defined (for a more detailed discussion of the "same" phenomena for $e_{C}$ and $c h$, see $\left.[2, \S 7]\right)$. We leave to the reader to check that $\lambda=e_{C}(\gamma) \in Q / Z$. The proof of (6.1) is now readily obtained directly from $(2.1)$, etc.

For a further study in the use and application of the Todd character we refer the reader to [8].

\section{Examples}

Our objective in this section is to show how certain results from the preceding discussions may be applied to construct some examples of a somewhat new and different type than those we have considered previously [6], [7], [13]. 
Example 1. Let $\eta \in \pi_{1}^{s} \cong Z_{2}$ and $\nu \in \pi_{3}^{s} \cong Z_{24}$ be elements of the stable stem of Hopf invariant 1. (These then generate the indicated cyclic groups.) The composition $\eta \circ \nu$ lies in the 4-stem which is zero [19]. Thus we may choose representative maps

$$
S^{2 k+1} \stackrel{\eta}{\longrightarrow} S^{2 k}, \quad S^{2 k+4} \stackrel{\nu}{\longrightarrow} S^{2 k+1}
$$

such that the composition

$$
S^{2 k+4} \stackrel{\nu}{\longrightarrow} S^{2 k+1} \stackrel{\eta}{\longrightarrow} S^{2 k}
$$

is null homotopic. Hence we may obtain a coextension [19, p. 13]

$$
\bar{\nu}: S^{2 k+5} \rightarrow S^{2 k} \cup_{\eta} e^{2 k+2}
$$

of $\nu$ and hence a space

$$
X=S^{2 k} \cup_{\eta} e^{2 k+2} \cup_{\bar{\nu}} e^{2 k+6} .
$$

Note that

$$
X / S^{2 k}=S^{2 k+2} \cup_{\Sigma \nu} e^{2 k+6}
$$

Thus we see that

$$
\widehat{H}_{i}(X ; Z) \cong \begin{cases}Z, & i=2 k, 2 k+2,2 k+6, \\ 0, & \text { otherwise },\end{cases}
$$

and that

$$
H^{2 k}\left(X ; Z_{2}\right) \stackrel{S q^{2}}{\longrightarrow} H^{2 k+2}\left(X ; Z_{2}\right) \stackrel{S q^{4}}{\longrightarrow} H^{2 k+4}\left(X ; Z_{2}\right)
$$

are isomorphisms. Now as $2 \eta=0$ we may obtain a map

$$
\tau: S^{2 k} \cup_{\eta} e^{2 k+2} \rightarrow S^{2 k}
$$

of degree 2 on the bottom sphere. The composition

$$
S^{2 k+5} \stackrel{\bar{\nu}}{\longrightarrow} S^{2 k} \cup_{\eta} e^{2 k+2} \stackrel{\tau}{\longrightarrow} S^{2 k}
$$

then represents the Toda bracket $\{2, \eta, \nu\}$ which lies in the 5-stem and hence must be null homotopic as $\pi^{s}{ }_{5}=0$ [19]. Thus we may extend $\tau$ to a map

$$
\tilde{\tau}: X \rightarrow S^{2 k}
$$

of degree 2 on the bottom sphere. 
Let $Y$ be the mapping cone of $\tilde{\tau}$. Then there is the cofibration

$$
X \underset{\tau}{\longrightarrow} S^{2 k} \underset{\theta}{\longrightarrow} Y
$$

Note that

$$
\bar{H}_{i}(Y ; Z) \cong \begin{cases}Z_{2}, & i=2 k, \\ Z, & i=2 k+3,2 k+7, \\ 0, & \text { otherwise },\end{cases}
$$

and

$$
H^{2 k}\left(Y ; Z_{2}\right) \stackrel{S q^{1}}{\longrightarrow} H^{2 k+1}\left(Y ; Z_{2}\right) \stackrel{S q^{2}}{\longrightarrow} H^{2 k+3}\left(Y ; Z_{2}\right) \stackrel{S q^{4}}{\longrightarrow} H^{2 k+7}\left(Y ; Z_{2}\right)
$$

are isomorphisms.

Let $\gamma=\left[S^{2 k}, \theta\right] \in \Omega_{2 k}^{U}(Y)$, and $b \neq 0 \in H^{2 k}\left(Y ; Z_{2}\right)$. Then

$$
S_{1} \beta b=S q^{2} S q^{1} b \neq 0, \quad S_{2} \beta b=S q^{4} S q^{2} S q^{1} b \neq 0 .
$$

Therefore according to (3.8)

$$
A(\gamma) \ni 2,\left[V^{2}\right]+d_{2},\left[V^{6}\right]+d_{6},
$$

where $d_{2}, d_{6}$ are decomposable elements of degree 2 and 6 respectively, all of whose mod 2 Chern numbers vanish. From Milnor's work [10] it follows that

$$
d_{2} \in(2), \quad d_{6} \in\left(2,\left[V^{2}\right]\right) .
$$

Therefore

$$
A(\gamma) \ni 2,\left[V^{2}\right],\left[V^{6}\right],
$$

and hence the girth of $A(\gamma)$ is at least 3. Inspection shows that

$$
\text { hom. } \operatorname{dim}_{\Omega_{*}^{U}} A(\gamma) \geq 2
$$

and hence by (4.1) we learn

$$
\text { hom. } \operatorname{dim}_{\Omega_{*}^{U}} \Omega_{*}^{U}(Y) \geq 3 .
$$

The space $Y$ is interesting because

$$
A(\gamma) \ni 2,\left[V^{2}\right],\left[V^{6}\right]
$$

despite the fact that the space $V(2)$ (see [13]) does not exist for the prime 2, that is, the attaching map 


$$
\lambda: S^{2 k+6} \rightarrow S^{2 k} \cup_{2} e^{2 k+1} \cup_{\bar{\eta}} e^{2 k+2}=V(1 / 2)
$$

representing the top cell satisfies

$$
\left[S^{2 k+6}, \lambda\right]=\left[V^{6}\right] \gamma .
$$

This is in striking contrast (as perhaps it should be) with the odd primary situation where $\left[V^{2 p^{2}-2}\right] \gamma$ is not a spherical bordism class on the space $V(1 / 2)$ $[6, \S 6]$.

The space $Y$ seems to be the "simplest" example of a space whose complex bordism module has projective dimension at least 3 . Note that the Thom map

$$
\mu: \Omega_{*}^{U}(Y) \rightarrow H_{*}(Y ; Z)
$$

is onto in dimension $2 k$ and hits twice the generator in dimension $2 k+2$ and eight times the generator in dimension $2 k+6$.

Example 2. The process which we employed in the previous example may be continued one more time. We let $\sigma \in \pi_{7}^{s} \cong Z_{16}+Z_{3}+Z_{5}$ be the element of order 16 with Hopf invariant one [19]. As before we may choose representative maps

$$
S^{2 k+1} \stackrel{\eta}{\longrightarrow} S^{2 k}, S^{2 k+4} \stackrel{\nu}{\longrightarrow} S^{2 k+1}, S^{2 k+12} \stackrel{\sigma}{\longrightarrow} S^{2 k+5}
$$

such that the composite

$$
S^{2 k+4} \stackrel{\nu}{\longrightarrow} S^{2 k+1} \stackrel{\eta}{\longrightarrow} S^{2 k}
$$

is null homotopic. Thus we may consider the composite

$$
S^{2 k+12} \stackrel{\sigma}{\longrightarrow} S^{2 k+5} \stackrel{\bar{\nu}}{\longrightarrow} S^{2 k} \cup_{\eta} e^{2 k+2} .
$$

Assertion. $\pi_{2 k+12}\left(S^{2 k} \cup_{\eta} e^{2 k+2}\right) \cong Z_{3}$ for $k$ large.

Proof. For $k$ large enough we are in the stable range and we have that the sequence

$$
\cdots \rightarrow \pi_{2 k+12}\left(S^{2 k}\right) \rightarrow \pi_{2 k+12}\left(S^{2 k} \cup_{\eta} e^{2 k+2}\right) \rightarrow \pi_{2 k+12}\left(S^{2 k+2}\right) \rightarrow \pi_{2 k+11}\left(S^{2 k}\right)
$$

is exact and isomorphic to the exact sequence

$$
\pi_{12}^{s} \rightarrow \pi_{2 k+12}\left(S^{2 k} \bigcup_{\eta} e^{2 k+2}\right) \rightarrow \pi_{10}^{s} \stackrel{\eta}{\longrightarrow} \pi_{11}^{s} .
$$

According to $[19$, p. 189] we have

$$
\pi^{s}{ }_{12}=0, \quad \pi^{s}{ }_{10} \cong Z_{2} \eta \mu+Z_{3} \beta_{1,3}, \quad \pi^{s}{ }_{11} \cong Z_{8} \zeta+Z_{9} \alpha^{\prime}{ }_{3,3}+Z_{7} \alpha_{1,7}
$$


and [19, Th. 14.1]

$$
\eta^{2} \circ \mu=4 \zeta \neq 0, \quad \eta \circ \beta_{1,3}=0
$$

and the result follows.

Thus for suitably large $k$ the 2-component of $\pi_{2 k+12}\left(S^{2 k} \cup_{\eta} e^{2 k+2}\right)$ is zero. As the composite

$$
S^{2 k+12} \stackrel{\sigma}{\longrightarrow} S^{2 k+5} \stackrel{\bar{\nu}}{\longrightarrow} S^{2 k} \cup_{\eta} e^{2 k+2}
$$

is an element of this 2-component we may conclude that this composite is null homotopic. Hence we obtain a coextension

$$
S^{2 k+13} \stackrel{\widetilde{\sigma}}{\longrightarrow} S^{2 k} \bigcup_{\eta} e^{2 k+2} \bigcup_{\bar{\nu}} e^{2 k+6}
$$

and we may form the complex

$$
A=S^{2 k} \cup_{\eta} e^{2 k+2} \cup_{\bar{\nu}} e^{2 k+6} \cup_{\widetilde{\sigma}} e^{2 k+14},
$$

note that

$$
\bar{H}_{i}(A ; Z) \cong \begin{cases}Z, & i=2 k, 2 k+2,2 k+6,2 k+14 \\ 0, & \text { otherwise }\end{cases}
$$

and that

$$
H^{2 k}\left(A ; Z_{2}\right) \stackrel{s q^{2}}{\longrightarrow} H^{2 k+2}\left(A ; Z_{2}\right) \stackrel{s q^{4}}{\longrightarrow} H^{2 k+6}\left(A ; Z_{2}\right) \stackrel{s q^{8}}{\longrightarrow} H^{2 k+14}\left(A ; Z_{2}\right)
$$

are iomorphisms.

Let now

$$
\tilde{\tau}: S^{2 k} \cup_{\eta} e^{2 k+2} \cup_{\bar{\nu}} e^{2 k+6} \rightarrow S^{2 k}
$$

be the map constructed in the previous example. Observe that the composite

$$
S^{2 k+13} \stackrel{\widetilde{\sigma}}{\longrightarrow} S^{2 k} \cup_{\eta} e^{2 k+2} \cup_{\bar{\nu}} e^{2 k+6} \stackrel{\widetilde{\tau}}{\longrightarrow} S^{2 k}
$$

lies in $\pi_{13}^{s} \cong Z_{3}$ (for $k$ large) and has order a power of 2 . Therefore this composite is null homotopic and hence we may extend $\tilde{\tau}$ to a map

$$
\hat{\tau}: A \rightarrow S^{2 k}
$$

of degree 2 on the bottom cell. Let now $B$ be the cofibre of $\hat{\tau}$ so we have the cofibration 


$$
A \underset{\hat{\tau}}{\longrightarrow} S^{\imath k} \underset{\hat{\theta}}{\longrightarrow} B
$$

Note that

$$
\tilde{H}_{i}(B ; Z) \cong \begin{cases}Z_{2}, & i=2 k \\ Z, & i=2 k+3,2 k+7,2 k+15, \\ 0, & \text { otherwise }\end{cases}
$$

and

$$
\begin{aligned}
H^{2 k}\left(B ; Z_{2}\right) \stackrel{s q^{1}}{\longrightarrow} H^{2 k+1}\left(B ; Z_{2}\right) \stackrel{s q^{2}}{\longrightarrow} H^{2 k+3}\left(B ; Z_{2}\right) \\
H^{2 k+15}\left(B ; Z_{2}\right) \stackrel{s q^{8}}{\longleftarrow} H^{2 k+7}\left(B ; Z_{2}\right)
\end{aligned}
$$

are all isomorphisms.

Let $\lambda=\left[S^{2 k}, \hat{\theta}\right] \in \Omega_{2 k}^{U}(B)$ and $a \neq 0 \in H^{2 k}\left(Y ; Z_{2}\right)$. Then

$$
\begin{aligned}
& S_{1} \beta a=S q^{2} S q^{1} a \neq 0, \\
& S_{2} S a=S q^{4} S q^{2} S q^{1} a \neq 0, \\
& S_{3} \beta a=S q^{8} S q^{4} S q^{2} S q^{1} a \neq 0 .
\end{aligned}
$$

Therefore according to (3.3),

$$
A(\lambda) \ni 2,\left[V^{2}\right]+d_{2},\left[V^{6}\right]+d_{6},\left[V^{14}\right]+d_{14},
$$

where $d_{2}, d_{6}, d_{14}$ are decomposable elements of degree 2,6, and 14 respectively, all of whose mod 2 Chern numbers vanish. From Milnor's work it follows that

$$
d_{2} \in(2), d_{6} \in\left(2,\left[V^{2}\right]\right), d_{14} \in\left(2,\left[V^{2}\right],\left[V^{6}\right]\right) .
$$

Therefore

$$
A(\lambda)=\left(2,\left[V^{2}\right],\left[V^{6}\right],\left[V^{14}\right]\right)
$$

and hence the girth of $A(\lambda)$ is at least 3. Inspection shows that

$$
\text { hom. } \operatorname{dim}_{\Omega_{*}^{U}} A(\lambda) \geq 3,
$$

and hence from (4.1) we learn

$$
\text { hom. } \operatorname{dim}_{\Omega_{*}^{U}} \Omega_{*}^{U}(B) \geq 4 \text {. }
$$


We believe that the space $B$ is by far the simplest space whose bordism module has projective dimension at least 4 .

Note that according to (6.3) the reduced Thom map

$$
\tilde{\eta}: Z[t] \otimes_{\Omega_{*}^{U}} \Omega_{*}^{U}(B) \rightarrow \tilde{k}_{*}(B)
$$

cannot be a monomorphism. Therefore the spectral sequence [6, Th. 10.2]

$$
E^{r} \rightarrow k_{*}(B), \quad E_{r, s}^{2}=\operatorname{Tor}_{r, s}^{\Omega_{*}^{U}}\left(Z[t], \Omega_{*}^{U}(B)\right)
$$

must be non-trivial, providing an example promised in the paragraph preceding $[6, \S 12]$.

As these examples exhaust the list of elements of Hopf invariant 1 in the stable stems [1], [11] we will be unable to proceed further along these lines.

Example 3. Let $p$ be a prime integer and denote by $\alpha_{1, p} \in \pi^{s}{ }_{2 p-3}$ an element of the stable $p$-component whose mod $p$ Hopf invariant is 1 [11], [18]. Fix a finite set of distinct primes $\left\{p_{1}, \cdots, p_{n}\right\}$ and let $q=p_{1} \cdots p_{n}$. Represent each $\alpha_{1, p_{i}}$ by a map

$$
\phi_{i}: S^{2 k+2 p_{i-3}} \rightarrow S^{2 k}, \quad i=1, \cdots, n .
$$

Observe that by choosing $k$ large enough each of the compositions

$$
S^{2 k+2 p_{i-3}-3} \stackrel{\phi_{i}}{\longrightarrow} S^{2 k} \stackrel{q}{\longrightarrow} S^{2 k}, \quad i=1, \cdots, n,
$$

may be assumed null homotopic. Let $X$ be the mapping cone of

$$
S^{2 k+2 p_{1}-3} \cdots S^{2 k+2 p_{n}-3} \stackrel{\phi_{1} \phi_{2} \cdots \phi_{n}}{\rightarrow-\cdots} S^{2 k}
$$

i.e.,

$$
X=S^{2 k} \cup_{\phi_{1}} e^{2 k+2 p_{1}-2} \cup_{\phi_{2}} \cdots \cup_{\phi_{n}} e^{2 k+2 p_{n}-2} .
$$

Note that

$$
\bar{H}_{i}(X ; Z)= \begin{cases}Z, & i=2 k, 2 k+2 p_{1}-3, \cdots, 2 k+2 p_{n}-3, \\ 0, & \text { otherwise }\end{cases}
$$

and that

$$
S_{1}=P_{p_{i}}^{1}: H^{2 k}\left(X ; Z_{p_{i}}\right) \rightarrow H^{2 k+2 p_{i}-2}\left(X ; Z_{p_{i}}\right)
$$

is an isomorphism for $i=1, \cdots, n$.

Since as noted previously $q$ annihilates each of the $\phi_{i}$ we may obtain an extension 


$$
\psi: X \rightarrow S^{2 k}
$$

of the map

$$
q: S^{2 k} \rightarrow S^{2 k}
$$

of degree $q$. Let $Y$ be the mapping cone of $\psi$ so that we have a cofibration sequence

$$
X \underset{\psi}{\longrightarrow} S^{2 k} \underset{\theta}{\longrightarrow} Y
$$

Denote by $\gamma \in \Omega_{2 k}^{U}(Y)$ the bordism class $\left[S^{2 k}, \theta\right]$. Then from (2.4) we obtain

$$
A(\gamma)=\left(q, \frac{q}{p_{1}}\left[V^{2 p_{1}-2}\right]+d_{1}, \cdots, \frac{q}{p_{n}}\left[V^{2 p-2}\right]+d_{n}\right),
$$

where $d_{i} \in \Omega_{2 p_{i-2}}^{U}$ is a decomposable class all of whose Chern numbers are divisible by $q / p_{i}, i=1, \cdots, n$. We may assume that $\left\{p_{1}, \cdots, p_{n}\right\}$ is arranged so that $p_{1}<p_{2}<\cdots<p_{n}$. Then assuming $n>1$ we have $p_{1}<q / p_{1}<q$. Therefore in order for all the $\bmod q$ Chern numbers of $d_{1}$ to vanish we must have according to [10] that $d_{1} \in(q)$. More generally we find that

$$
d_{i} \in\left(\frac{q}{p_{1} \cdots p_{i-1}},\left[V^{2 p_{1}-2}\right], \cdots,\left[V^{2 p_{i-1}-2}\right]\right), \quad i>1,
$$

and thus inductively that

$$
A(\gamma)=\left(q, \frac{q}{p_{1}}\left[V^{2 p_{1}-2}\right], \cdots, \frac{q}{p_{n}}\left[V^{2 p_{n}-2}\right]\right) .
$$

We note also that

$$
\bar{H}_{i}(Y ; Z)= \begin{cases}Z_{q}, & i=2 k \\ Z, & i=2 k+2 p_{j}-1 ; j=1, \cdots, n, \\ 0, & \text { otherwise }\end{cases}
$$

The space $Y$ is closely related to and slightly generalizes the spaces $V(1 / 2)$ of [13].

Example 4. Let $a \in H^{2}(C P(n) ; Z)$ be the canonical class. Then for a suitable integer $d(n)$ there exists a map

$$
f_{n}: \Sigma^{2 k-2} C P(n) \rightarrow S^{2 k}
$$

of degree $d(n)$ on the bottom cell, i.e., with 


$$
f_{n}^{*} i=d(n) a
$$

for large $k$. For small values of $n$ one may compute with the aid of [19] that

$$
d(1)=1, d(2)=2, d(3)=24, d(4)=24 \text {, etc. }
$$

Note that according to our computations in $\S 1$ of the value of $S_{i}$ on a 2-dimensional class we will have for any prime $p$

$$
S_{i} a=a^{p i} \in H^{2 i}\left(C P(n) ; Z_{p}\right)
$$

and hence

$$
S_{i}(a) \neq 0 \in H^{*}\left(C P(n) ; Z_{p}\right)
$$

whenever $p^{i} \leq n$.

Let now $D(n)$ be the mapping cone of $f_{n}$ so that we have a cofibration sequence

$$
\Sigma^{2 k+2} C P(n) \underset{f_{n}}{\longrightarrow} S^{2 k} \underset{h}{\longrightarrow} D(n) .
$$

Let $\gamma_{n}=\left[S^{2 k}, h\right] \in \Omega_{2 k}^{U}(D(n))$. Then according to (2.4) we find

$$
\begin{aligned}
& A\left(\gamma_{n}\right) \ni d(n), \\
& \quad \frac{d(n)}{2}\left[V^{2}\right]+d_{2,2}, \cdots, \frac{d(n)}{2}\left[V^{2 i-2}\right]+d_{2,2 i-2}, \quad 2^{i}-2 \leq 2 n, \\
& \quad \frac{d(n)}{3}\left[V^{4}\right]+d_{3,4}, \cdots, \frac{d(n)}{3}\left[V^{2\left(3^{j}-1\right)}\right]+d_{3,2\left(3^{j}-1\right)}, \quad 3^{j}-1 \leq n, \\
& \quad \frac{d(n)}{p}\left[V^{2 p-2}\right]+d_{p, 2 p-2}, \cdots, \frac{d(n)}{p}\left[V^{2\left(p^{l}-1\right)}\right]+d_{p, 2\left(p^{l}-1\right)}, \quad p^{l}-1 \leq n,
\end{aligned}
$$

where $p$ is the largest prime no greater than $n$, and the $d$ 's are decomposables with all Chern numbers divisible by $d(n)$.

For example:

$$
A\left(\gamma_{2}\right)=\left(2,\left[V^{2}\right]\right), A\left(\gamma_{3}\right)=\left(24,12\left[V^{2}\right], 6\left[V^{4}\right], 12\left[V^{6}\right]+d_{2,6}\right), \text { etc. }
$$

(The arguments needed to eliminate the decomposable tails are similar to that in Example 3 and left to the reader.)

The computation of $d(n)$ and of a lower bound for the projective dimension of $A\left(\gamma_{n}\right)$ would be of considerable interest. Note that from (4.3) we learn that we certainly have hom. $\operatorname{dim}_{\Omega_{*}^{U}} \Omega_{*}^{U}(D(n))>1$. 


\section{References}

[1] J. F. Adams, On the non-existence of elements of Hopf invariant one, Ann. of Math. 72 (1960) 20-104.

[2] - On the groups J(X)-IV, Topology 5 (1966) 21-71.

[3] M. F. Atiyah \& F. Hirzebruch, Riemann-Roch theorems for differentiable manifolds, Bull. Amer. Math. Soc. 65 (1959) 276-281.

[ 4 ] H. Cartan \& S. Eilenberg, Homological algebra, Princeton University Press, Princeton, 1956.

[ 5 ] P. E. Conner \& E. E. Floyd, The relation of cobordism to K-theories, Lecture Notes in Math., No. 28, Springer, Berlin, 1966.

[6] P. E. Conner \& L. Smith, On the complex bordism of finite complexes, Inst. Hautes Études Sci. Publ. Math. No. 37 (1969) 117-221.

[ 7 ] _ - Homological dimension of complex bordism modules, Proc. Conf. Topology (Georgia), to appear.

[8] - Bordism invariants of three cell complexes, to appear.

[9] J. W. Milnor, The Steenrod algebra and its dual, Ann. of Math. 67 (1958) 150171.

[10] _ - On the cobordism ring * and a complex analog, Amer. J. Math. 82 (1960) 505-521.

[11] N. Shimado \& T. Yamanoshita, On triviality of the mod p Hopf invariant, Japan J. Math. 31 (1961) 1-25.

[12] L. Smith, An application of complex bordism to the stable homotopy groups of spheres, Bull. Amer. Math. Soc., to appear.

[13] —- On realizing complex bordism modules, Amer. J. Math., to appear.

[14] - On characteristic numbers for almost complex manifolds with framed boundaries, Topology, to appear.

[15] _ - On the relation of connective K-theory to integral homology, Proc. Cambridge Phil. Soc., to appear.

[16] _- On annihilator ideals of framed complex bordism classes, to appear.

[17] R. E. Stong, Notes on cobordism theory, Princeton University Press, Princeton, 1969.

[18] H. Toda, p-primary components of homotopy groups. I-IV, Mem. Univ. Kyoto 31 (1958) 129-142, 143-160, 191-210, 321 (1959) 297-332.

[19] - Composition methods in the homotopy groups of spheres, Annals of Math. Studies, No. 49, Princeton University Press, Princeton, 1962.

[20] _- A survery of homotopy theory, Sûgaku 15 (1963/64) 141-155.

UNIVERSITY OF VIRGINIA 